\title{
صورة الحضارة المصرية القديمة في السينما الأمريكية منذ بداية النصف الثاني من القرن العشرين المئن (دراسة للمناظر ومواقع التصوير)
}

ريهام محمد هلال

الملخص:

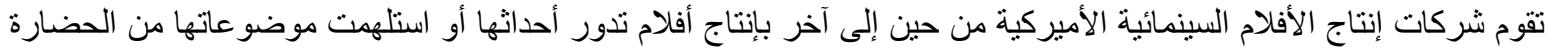

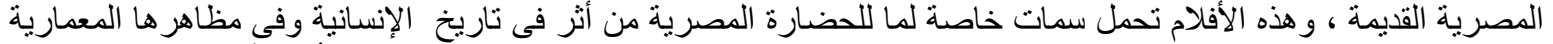

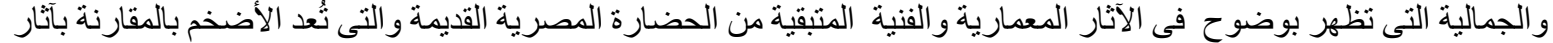

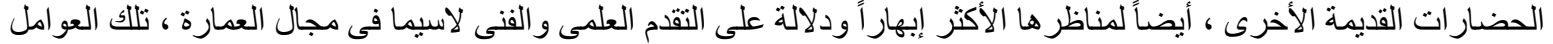

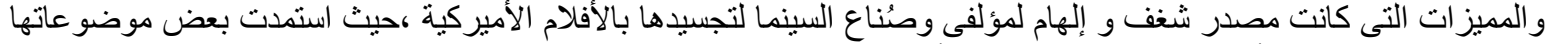

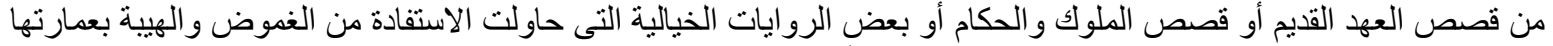

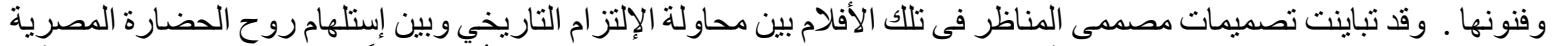

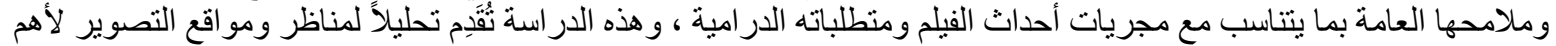

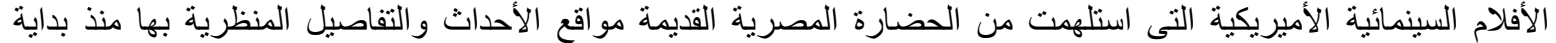

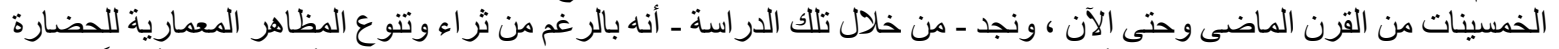

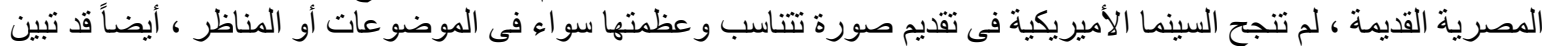

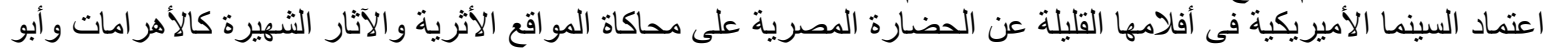

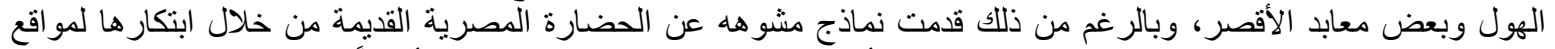

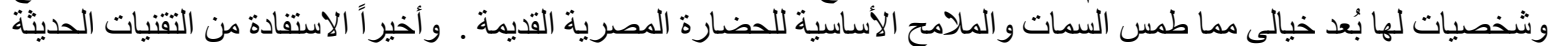

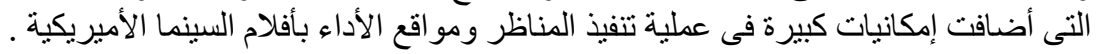

الكلمات الدالة: الحضارة الدصرية القديمة ؛ السينما الأمبيريكية ؛ الدناظر السينمائية

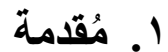

إن الآثار التىى بقيت من الحضارة المصرية القديمة وبعد آلاف السنين هى الأضخم بالمقارنة بآثار الحضارات القديمة الأخرى

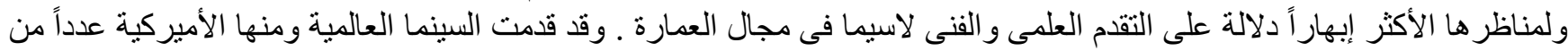

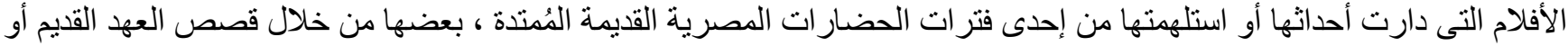

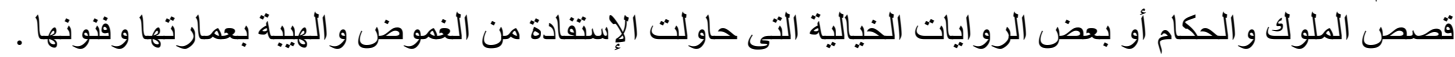

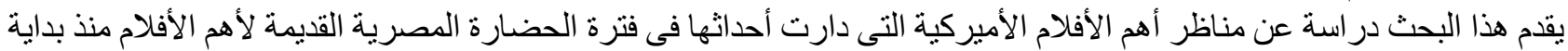

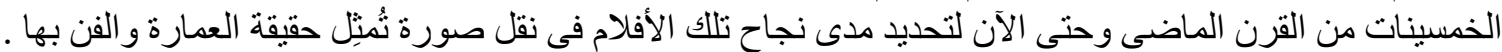

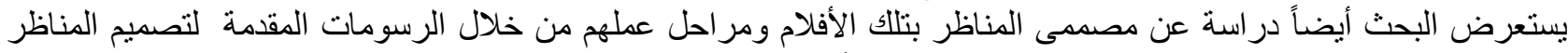
ومو اقع الأداء وكيفية تنفيذ و إنشاء تللك المناظر وتجهيز ها استعداداً لمرحلة التصوير ، والتقنيات و الأساليب الحديثة المستخدمة فى تصميم 


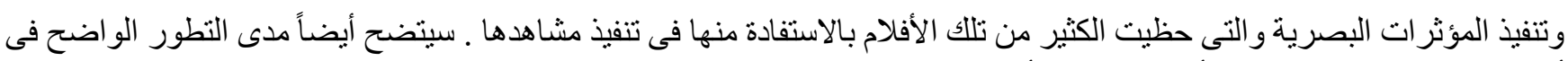

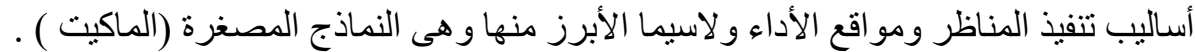

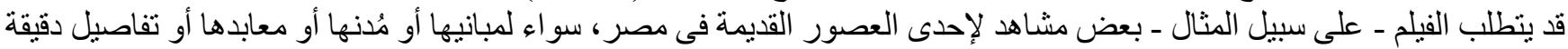

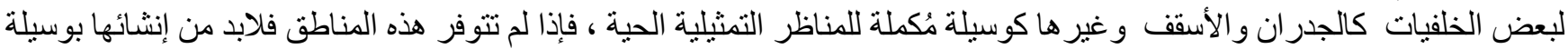

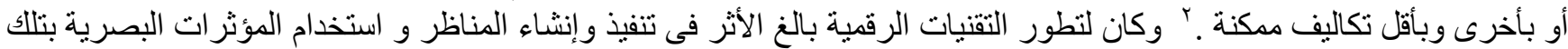

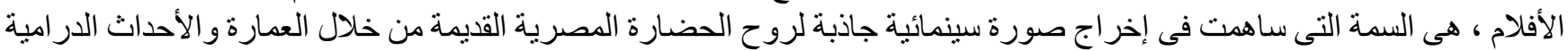

تزخر الحضارة المصرية القديمة بطابع معمارى ذى أصالة تاريخية من طرز وتصميمات لامثيل لها فى التميزو التفرد ، شكلت الملامح

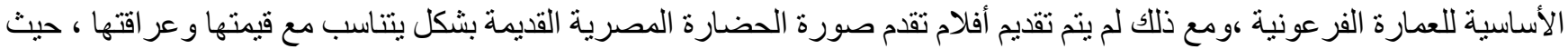

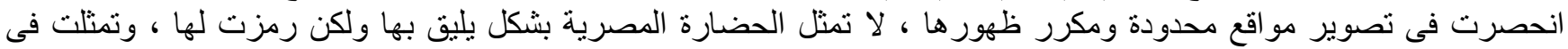

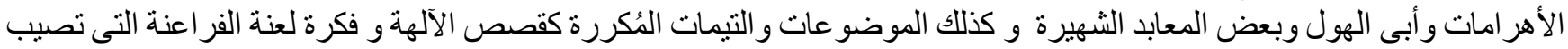

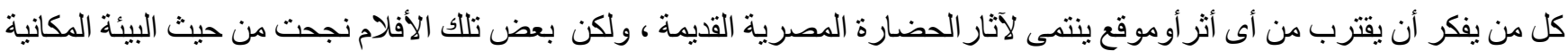
وتحقيقها للمتطلبات الدرامية والجمالية للأحداث بسيناريو الفيلم ـ وقد حدد هذا البحث أهم تلك الأفلام بداية من فيلم

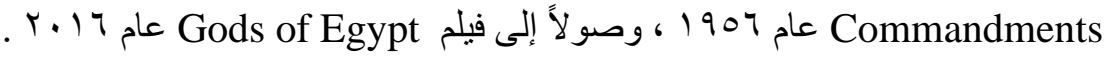

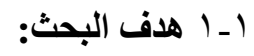

عمل در اسة تحليلية لمناظر ومو اقع الأداء فى الأفلام السينمائية الأمريكية التى استلهمت من الحضارة المئية المصرية ، لتحديد مدى نجاح تصميمات

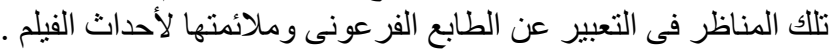

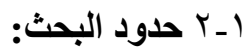
يقدم البحث در اسة وتحليل لمناظر بعض أفلام السينما الاميريكية المستلهمة من الحضارة المصرية القديمة منذ النصف الثانى من القرن العشرين وحتى وقتنا هذا .

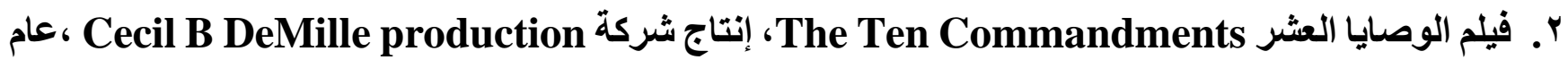

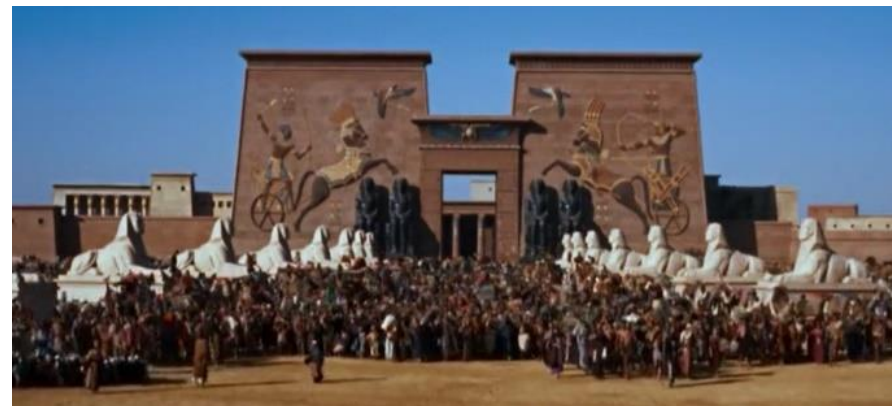

شكل ا ، منظر واجهة مدخل قصر سيتى كما ظهر بالفيلم ونظهر

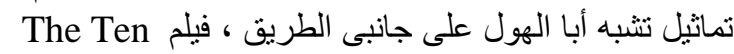
Commandments
r*Cecil B. DeMille 1904

هو فيلم يستند للقصة التور اتية لحياة النبى موسى عليه السلام ، وقد تم تصويره في مصر وفى جبل سيناء ويشبه جزيرة سيناء

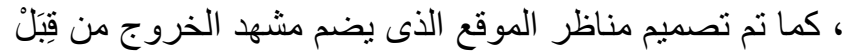

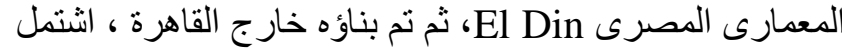

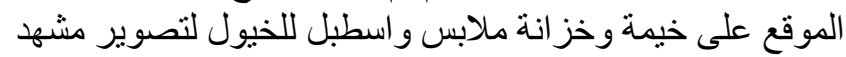

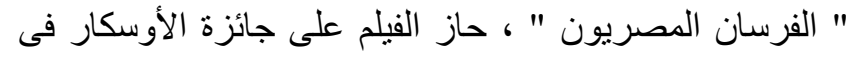

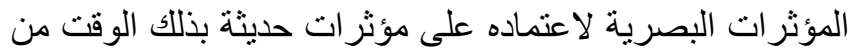

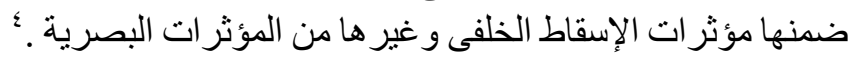

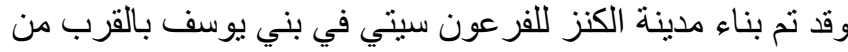

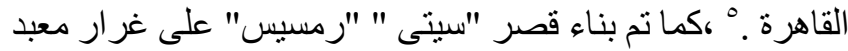
آهون فى الكرنك فى الأقصر ، ولكن تم استبدال تماثيل الكباش على عانى جانبى الطريق بتماثيل تشبه أبو الهول . 


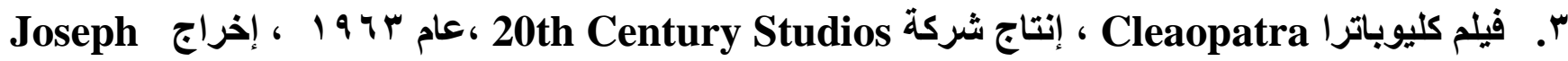 John Decuir* تصميم مناظر : جون ديكيور ، ميرة ، L. Mankiewicz*}

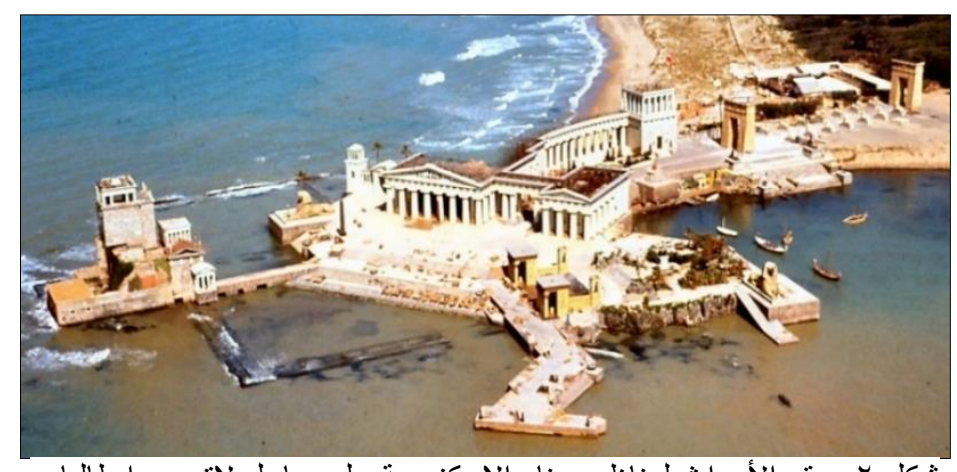

شكل r موقع الأحداث لمناظر ميناء الإسكندرية على ساحل لاتيوم ، إيطاليا ،

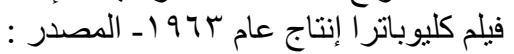
http://bigerboat.com/indexfx/?p=3777

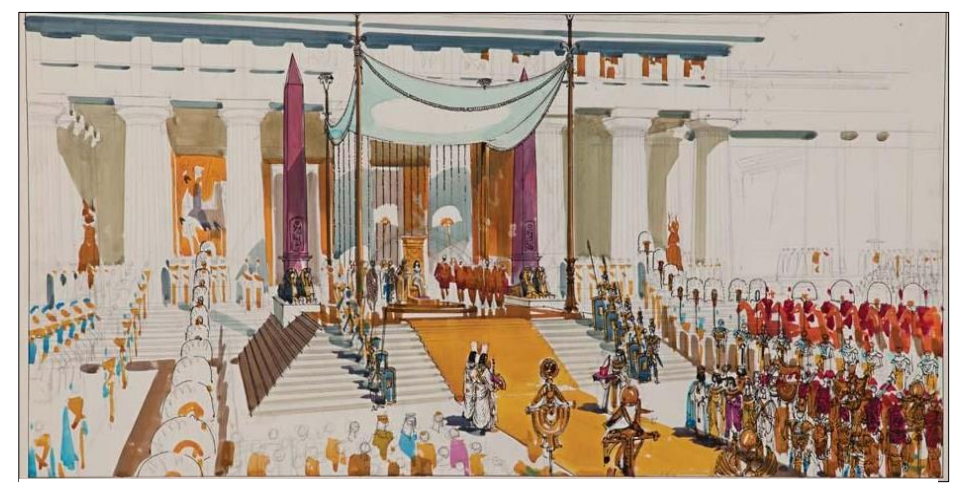

شكلץ رسم الدر اسة المبئية لفكرة تصميم مدخل قصر كليو باتر ا، لمصمح

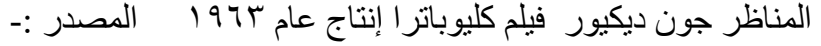
/https://www.pinterest.com/pin/312507661628025474

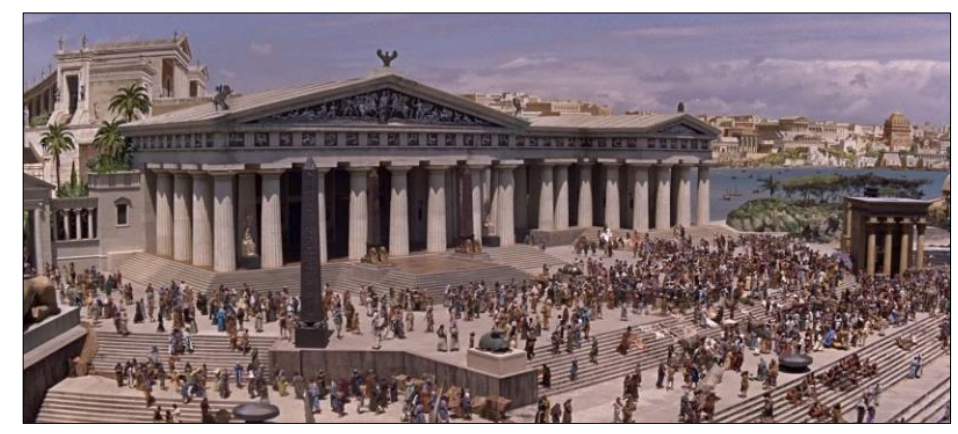

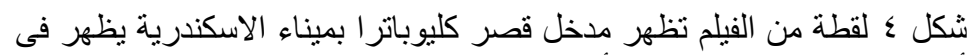

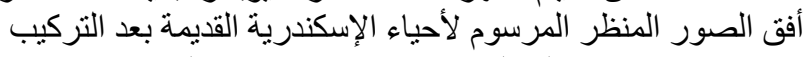

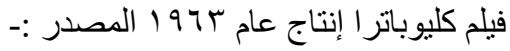
http://bigerboat.com/indexfx/?p=3777

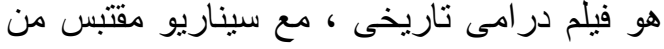
كتاب ( The life \& times of Cleopatra) لـ كارلو ماريا فر انزيرو Carlo Maria Franzero و ومن رو ايات

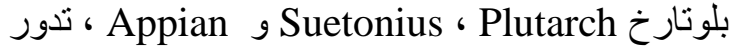
أحداث الفيلم فى الفترة مابين عامى 48 و و 30 قبل

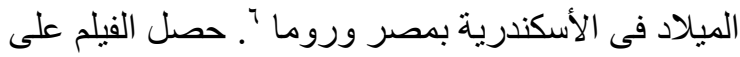

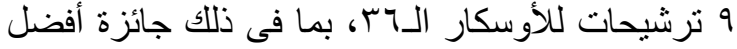

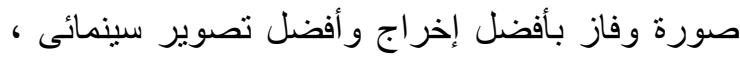
و أفضل مؤثرات بصرية و أفضل تصميم أزياء - إيرين

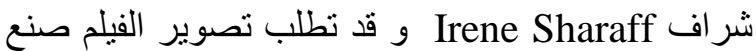

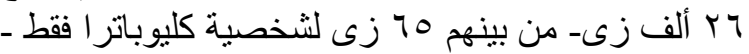

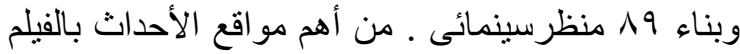

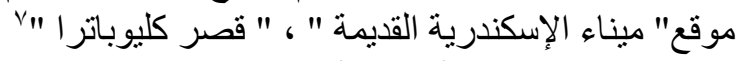

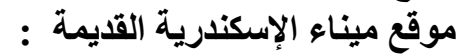

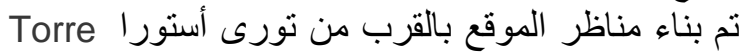

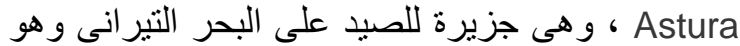
المكان الذى تصل إليه بارجة كليوباتر الطبقاً لأحداث الفيل الفيلم

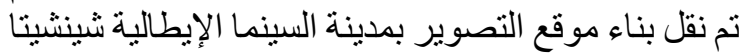
هائه Cinecitta خاص فى مدينة أنزيو جنوب إيطاليا ، لإظهار معركة الإنة

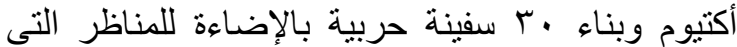

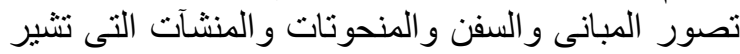
^ل للمعالم التاريخية المبنية

يتضمن موقع ميناء الإسكندرية مظاهر معمارية تحمل سمات العمارة الإغريقية متمثلة فى المعبد و الأعمدة الإعة

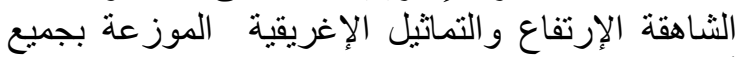

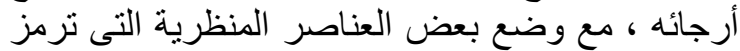

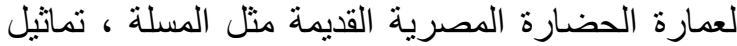
مستلهمة من أبى الهول ، وقد كانت التهرية التركيبة التى أظهرت

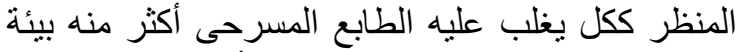

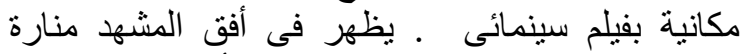

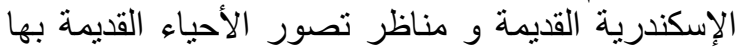

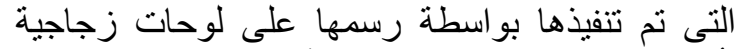

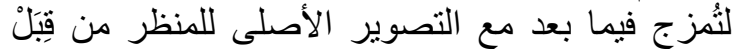
الفنان المصور جوزيف ناتانسون Joseph Natanson من لأنطي

* جوزيف ليو مانكيفيت Joseph Leo Mankiewicz (

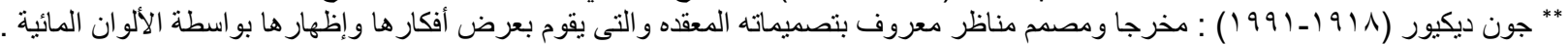

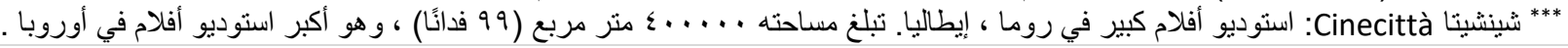




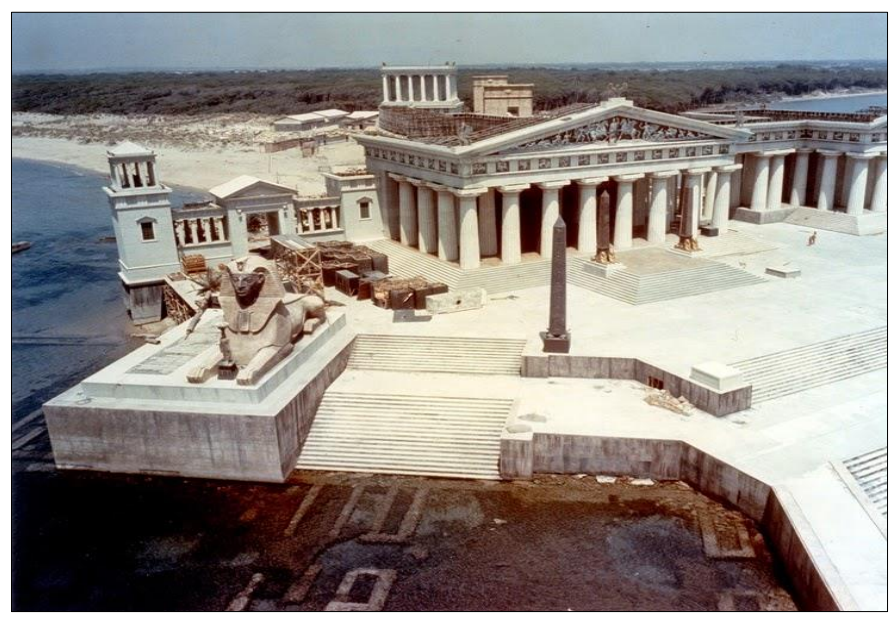

يظهر فى الشكل رقم (0) موقع تصوير ميناء الإسكندرية القديمة أثناء

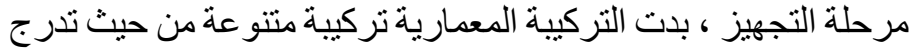

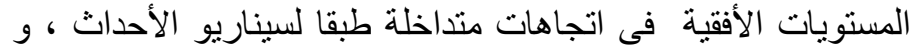

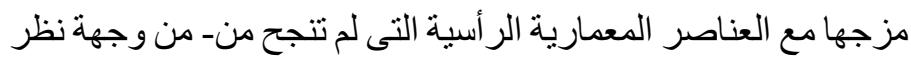

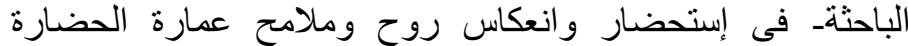

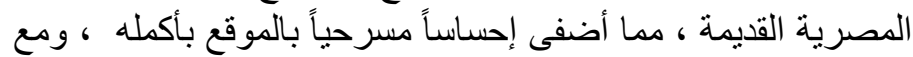
ذللك هذا لا ينقص شىء من القيمة الجمالية التى تحققت فى البيئة المكانية

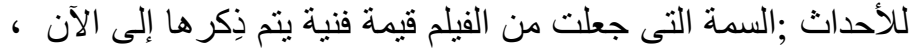

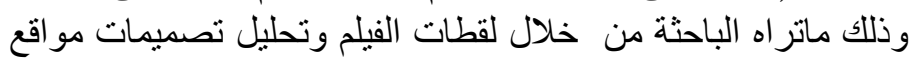

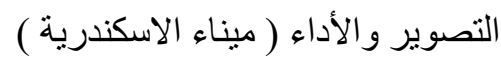
موقع قصر كليوباتر ا من الداخل :

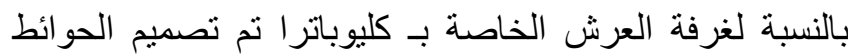

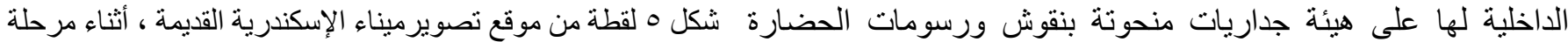

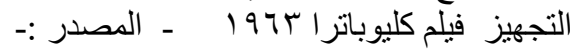
http://bigerboat.com/indexfx/?p=3777

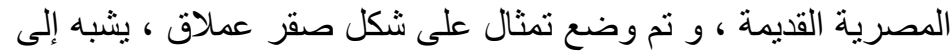

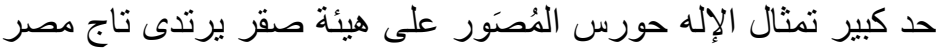

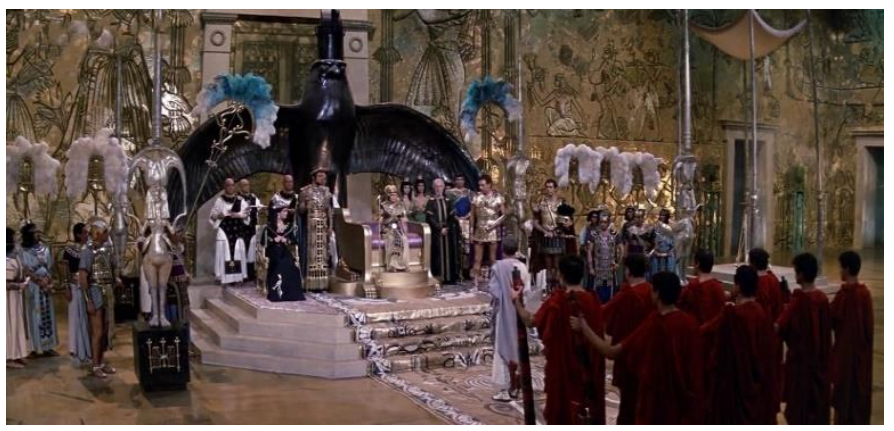

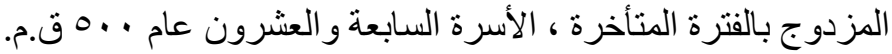

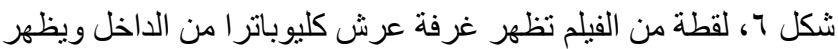

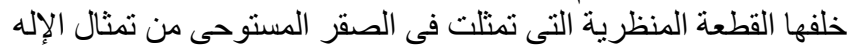

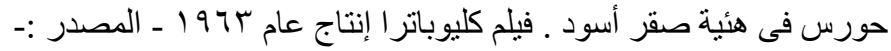
لقطة من الفيلم

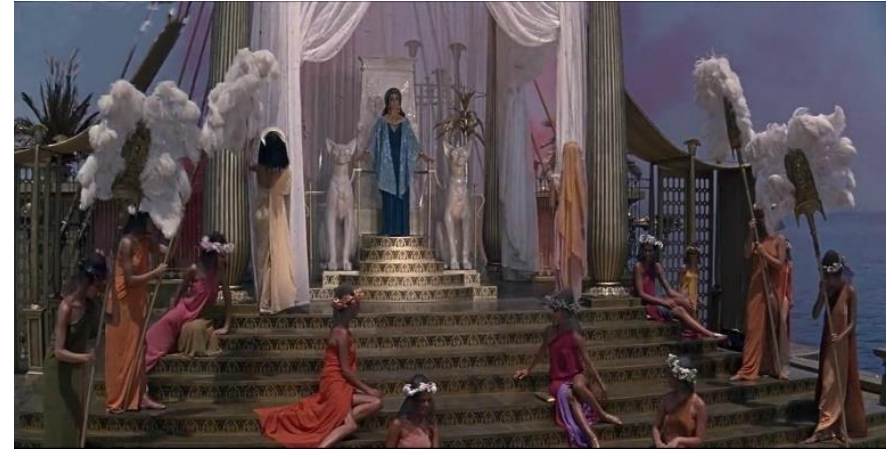

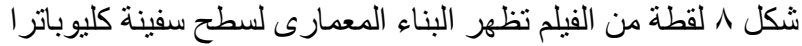
فيلم كليو باترا إنتاج عام بـ 197 المصدر :- لقطة من الفيلم

\section{موقع سطح سفينة كليوباترا :}

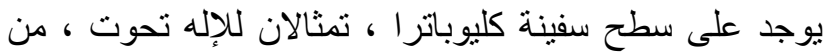
الممكن أن تكون هذه هي نفس المجسمات النحتية التى استخدمت في

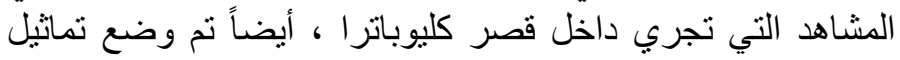

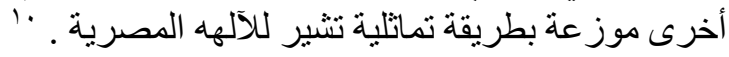

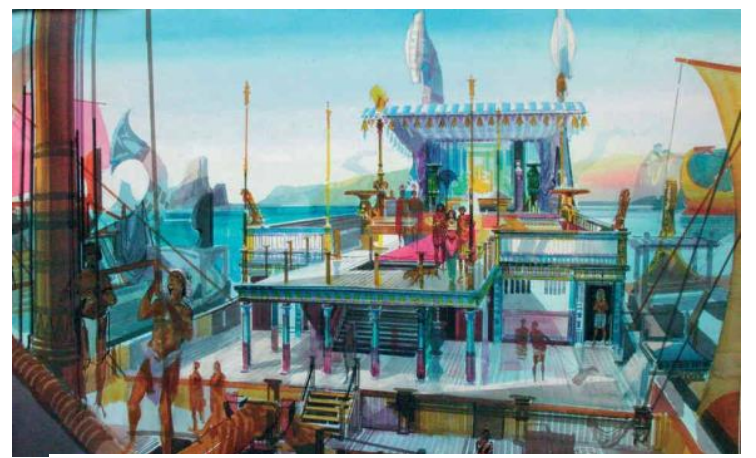

شكل V الدر اسة المبدئية لفكرة تصميم سطح سفينة ولكن

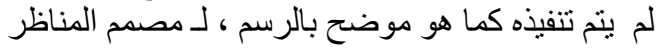

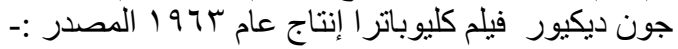
https://www.pinterest.com/pin/41552750310 $\underline{14615215}$ 


\section{SJFA}

Scientific Journal of the Faculty of Fine Arts Alexandria University

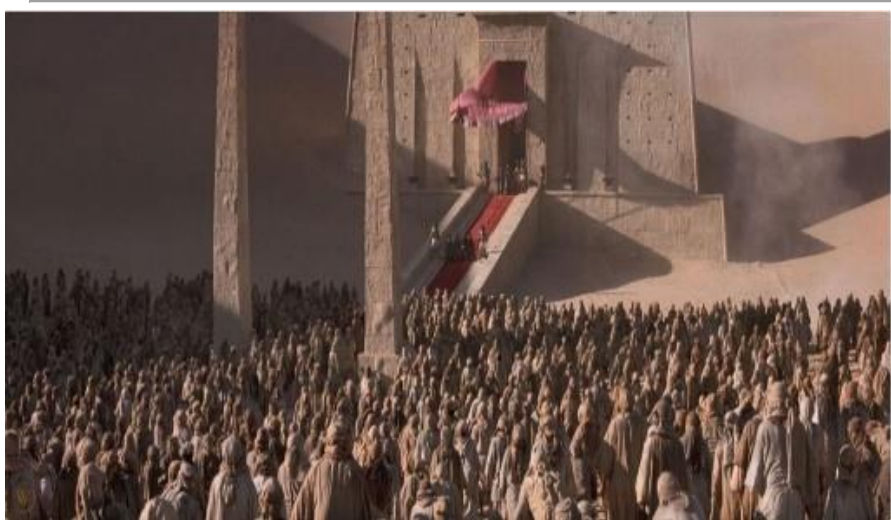

شكل 9 ، مدخل الهرم كما ظهر فى الفيلم ، فيلم stargate إنتاج عام ع 99 إــالمصدر : لقطة من الفيلم

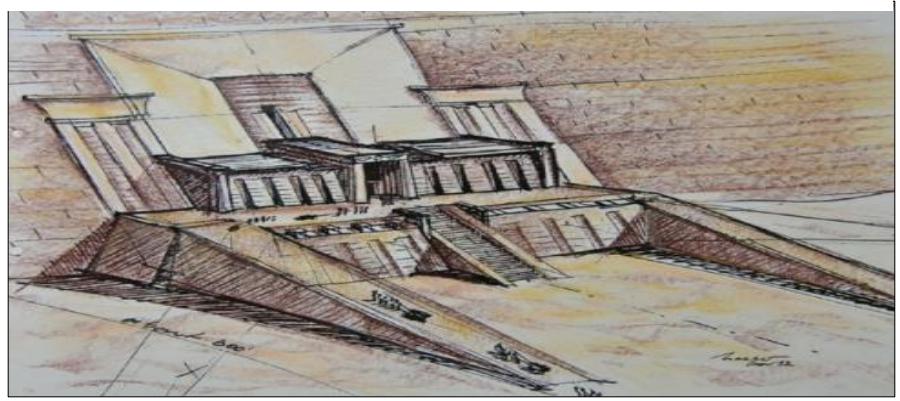

شكل •ا الدر اسة المبدئية التى تمثل مدخل الهرم المستوحى من معبد

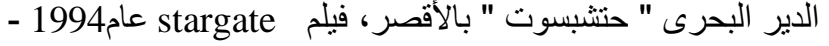

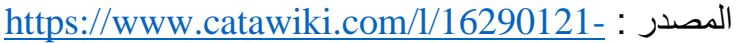

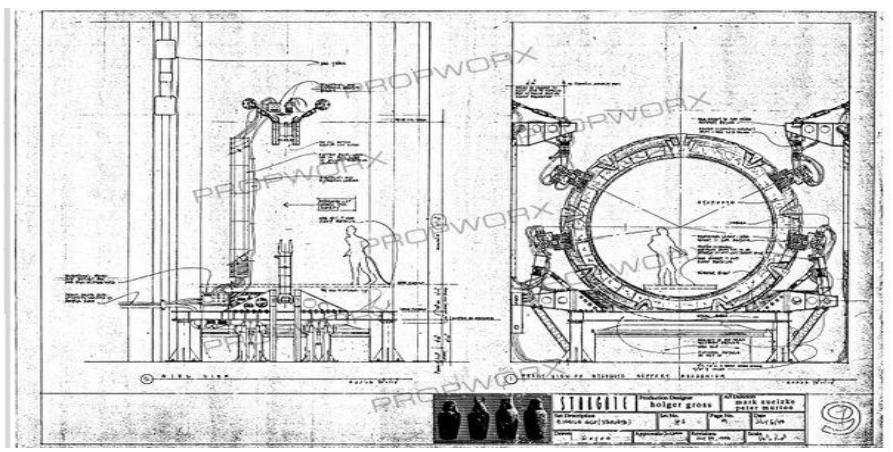

شكل I ال، الرسم الهندسى لتصميم بوابة Stargate بفيلم Stargate عام https://www.syfy.com/syfywire/build--: بصئ 199 your-own-stargate-using-the-original-1994-movieblueprints
فيلم Stargate-Goldwyn- ، إنتاج Mayer Roland Emmerich هولجر جروس Holger Gross

يعتبر فيلم Stargate من أفلام الخيال العلمى ، وتدور

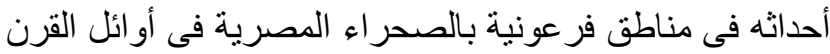
العشرين ، تعتمد أحداثه على الحضارة المصرية القديمة والآثار

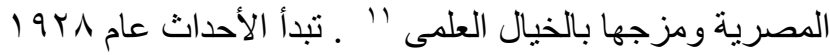
حول العثور على قطعة أثرية قديمة اكتشفت فى أهر امات الجيزة

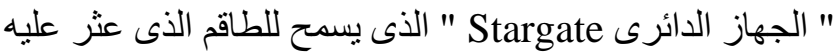

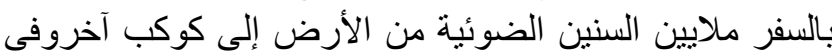

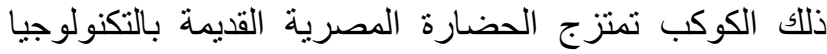

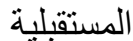
قام مصمم المناظربتصميم هذا العالم الخيالى لمواقع الأداء بأحداث الفيلم ، ومن أجل تحرى الدقة التاريخية تم الاستعانة بعلماء المصريات للمساعدة فى الكتابة الهيروغليفية التى التى

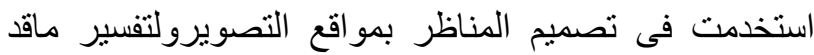
تبدو عليه اللغة المصرية القديمة. ، تشمل مناظر الفيلم تحول

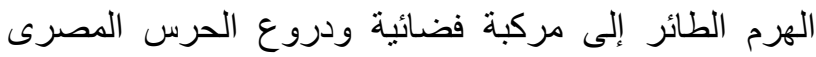
ويظهر بالأشكال التالية دراسات مبدئية للعديد من التصائية

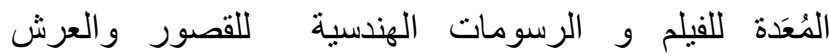

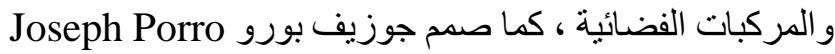

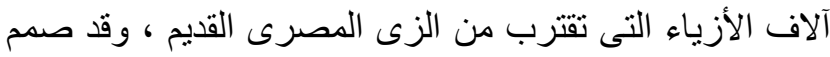

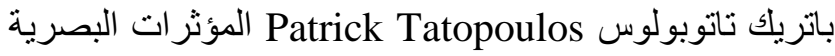

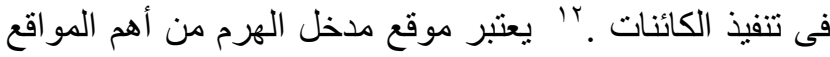

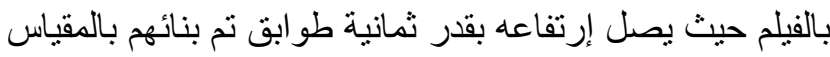

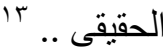

تنفيذ المنظر بمشهد فتح بوابة Stargate طبقاً لقصة الفيلم هى البوابة التي تستخدم الأبر اج كإحداثيات

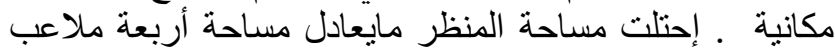

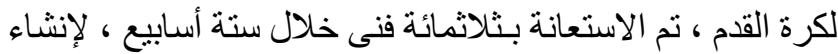

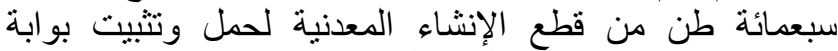
Stargate

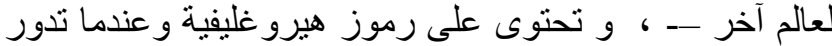

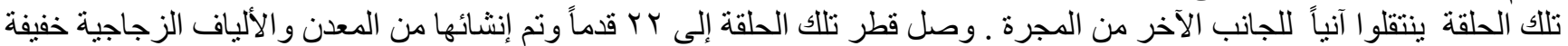

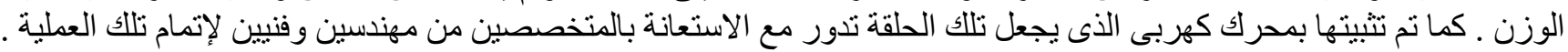

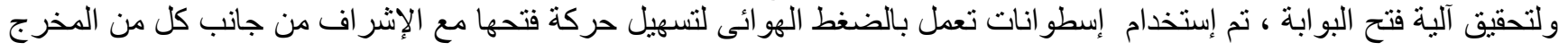

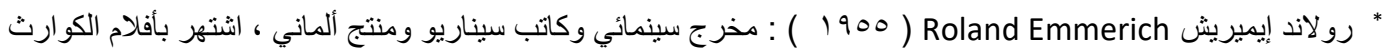

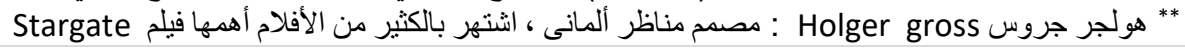




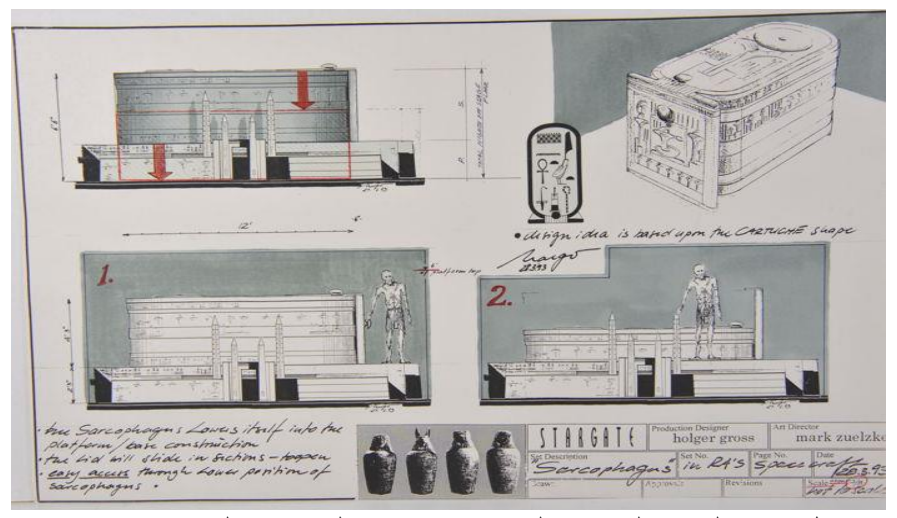

Stargate شكل r ا، الرسم الهندسى لتصميم مقبرة الإله رع ع فيلم

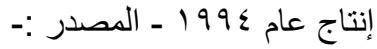

https://www.catawiki.com/l/16290161-stargatesarcophag-5-original-drawings

ومصدم المناظر و المسؤول عن المؤثرات البصرية ـ ـتم بناء

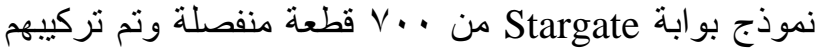

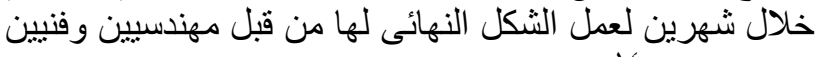

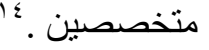
تنفيذ المنظربمشهد ظهور رع من مقبرتهه :

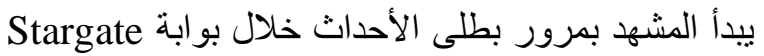

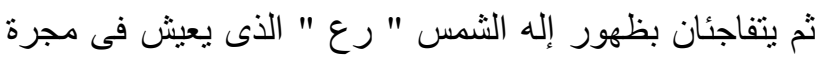

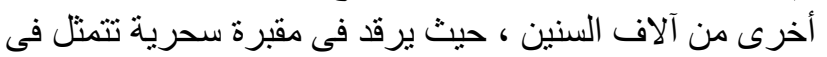

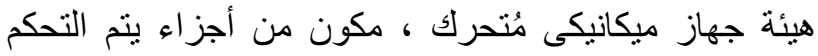

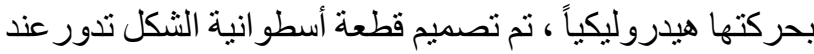

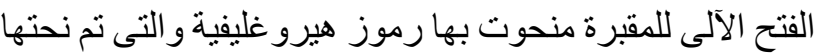

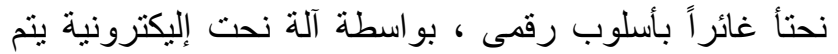

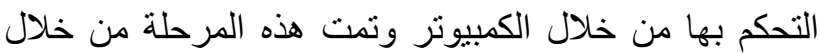

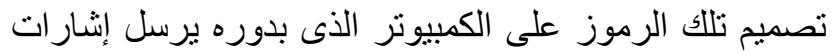

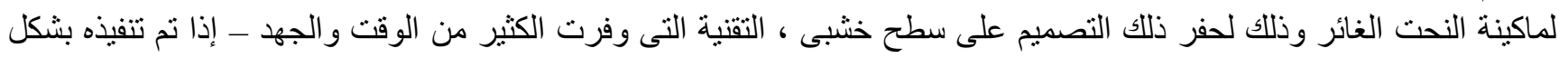

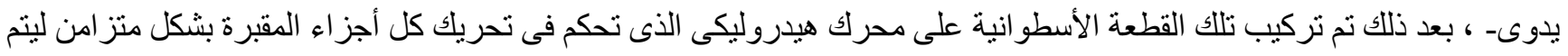

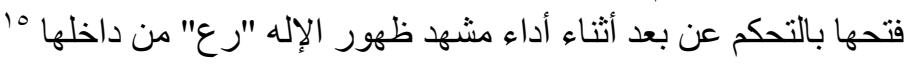

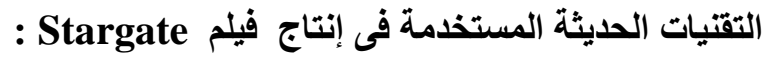

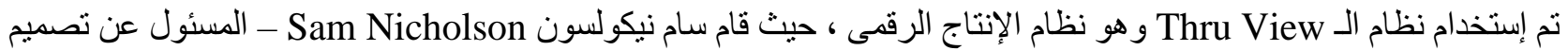

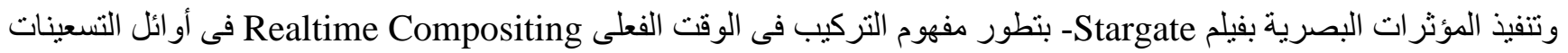

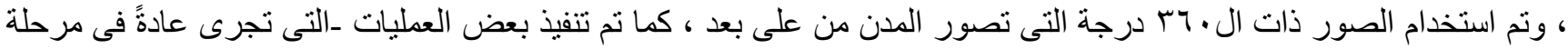

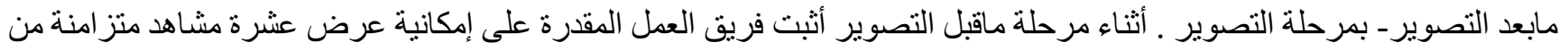
نوع لقطات 8K على أربعين شاشة

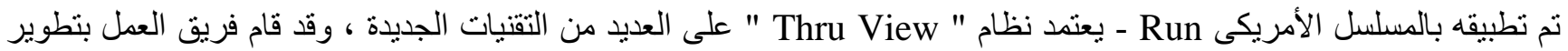

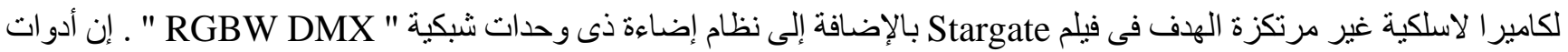

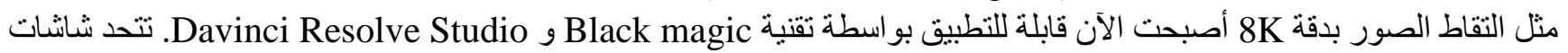

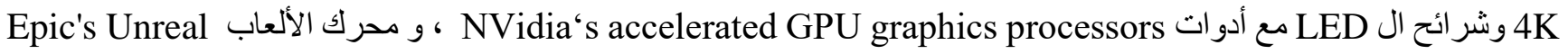
4 مع أدو ات Blackmagic Design لجعل نظام "Thru View" ممكنًا لفيلم Stargate ـ يتمثل التحدى في الجمع بين هذه التقنيات

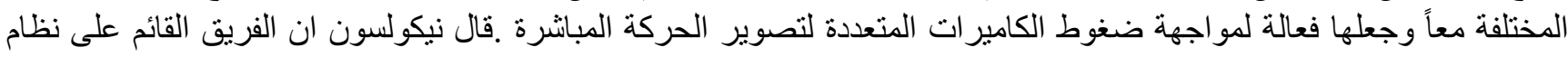

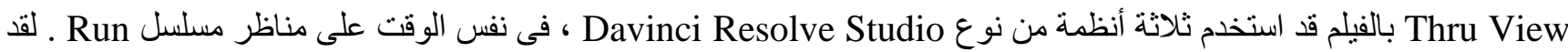

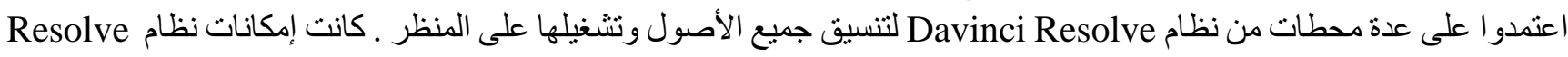

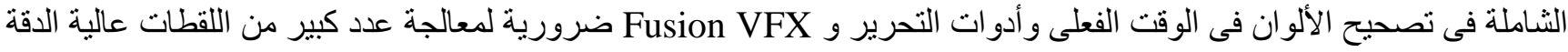

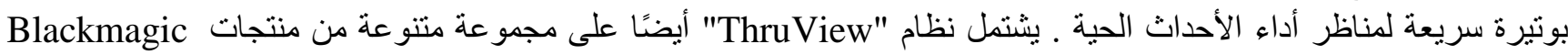

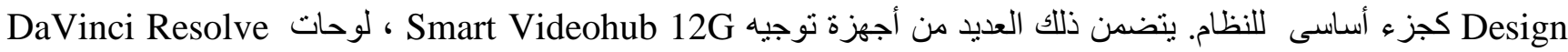
C UltraStudio 4K Extreme ، Micro ، محول معايير Teranex AV ، و مجمو عة من المحو لات الصغيرة ، بالإضافة إلى محول ATEM Constellation 8K

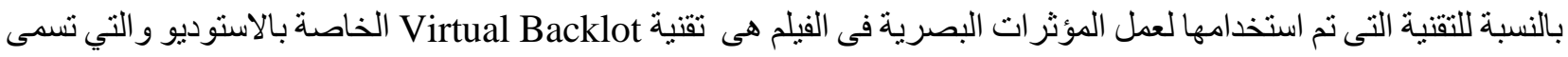

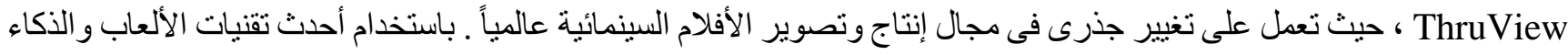

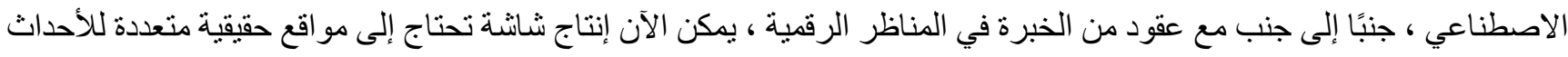

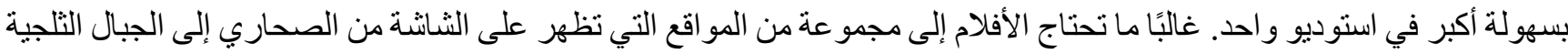
إلى السو احل أو شو ارع المدينة ، و التي يمكن الآن تصوير ها جميعًا في مكان و احد يتم التحكم فيه باستخدام نظام ThruView. 


\section{SJFA}

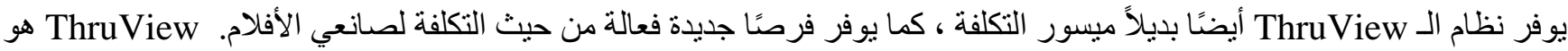

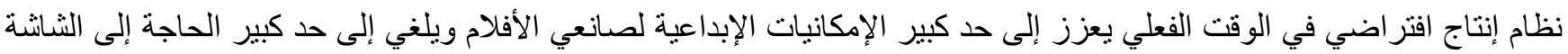

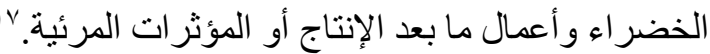

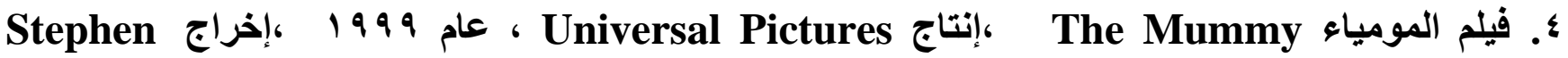
** Allan Cameron Sommers ، تصميم المناظر للمصمم آلان كاميرون ،

هو فيلم خيالى ويُعَدْ إعادة إنتاج لفيلم تم إنتاجه عام 1932 يحمل نفس الإسم ، وتدور أحداثه بين الفترة ، الفرعونية عام 1290 فئل 126 فبل الميلاد فى عهد الفرعون سيتى الأول والفترة الأخرى عشرينات القرن العشرين 1926 نظهر بأحداث الفيلم مدينة طيبة المصرية

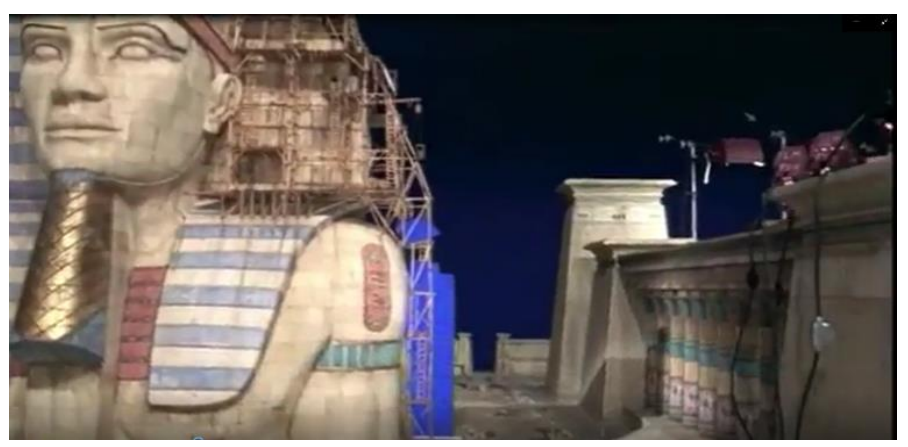

شكل سا 1 ، النموذج المصغر ثلاثى الأبعاد لمدينة طيبة، ويظهر تمثال

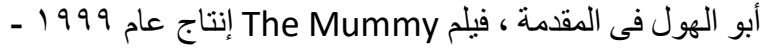

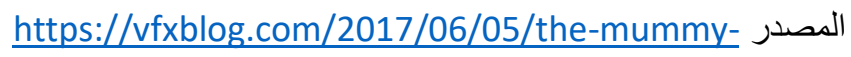
$\underline{1999-v f x}$

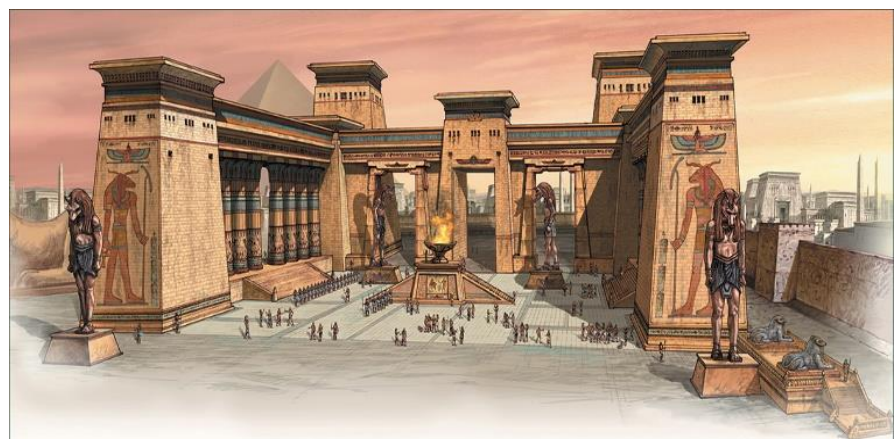

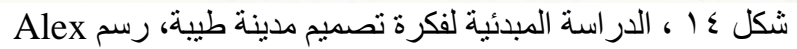

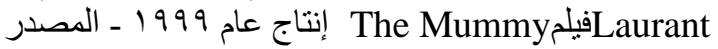
https://vfxblog.com/2017/06/05/the-mummy-1999-

$\underline{\text { vfx }}$
القديمة Ancient Thebes وتُظِيهر عر اقة الحضارة المصرية

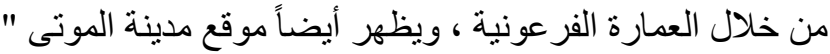

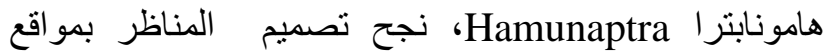

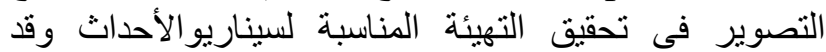

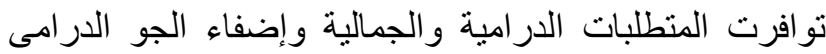

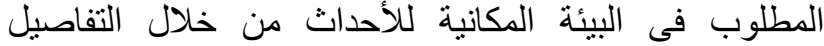

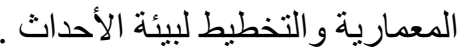

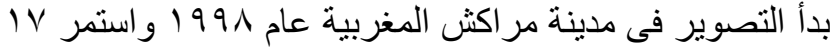

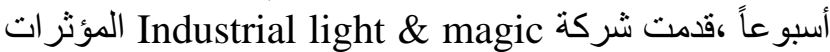

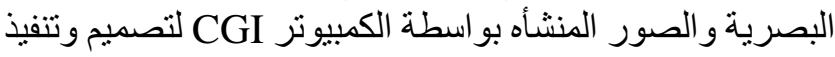
نموذج المومياء الرقمى ، وقد نتج عن نجاح الفيلم عمل سلسلة أفلام

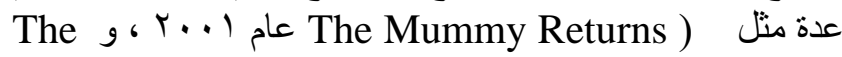

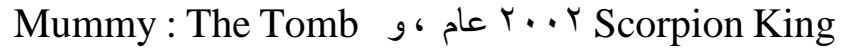
) عام ا.... of Dragon Emperor

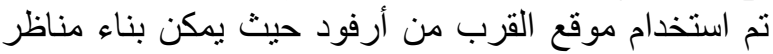

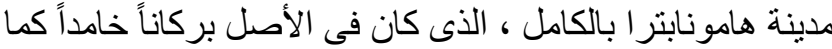

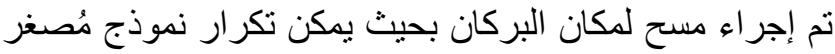

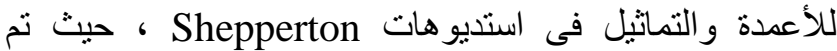
تصوير جميع المشاهد التى تشنتمل على ممرات تحت الته الأرض

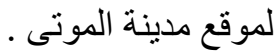

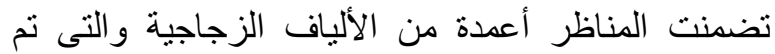

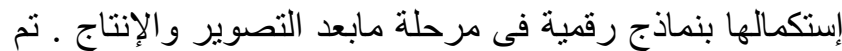

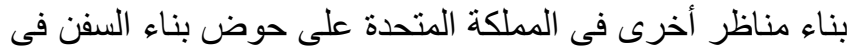
تشاثام Chatham Dockyard و التى تم مضاعفة مناعاحته

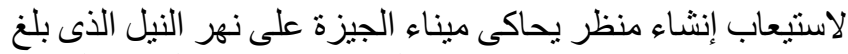

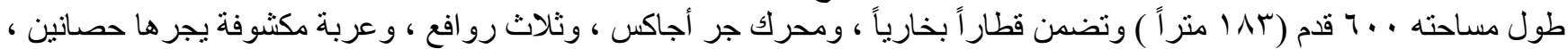

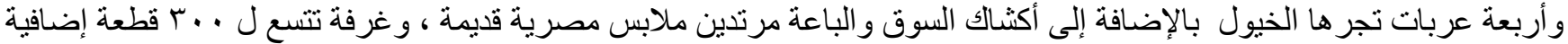
بالملابس . أما بالنسبة لإستخدام المؤثرات البصرية ، بدأ جون بيرتون John Berton *** ـ المسؤول عن تصميم وتتفيذ المؤثرات البصرية

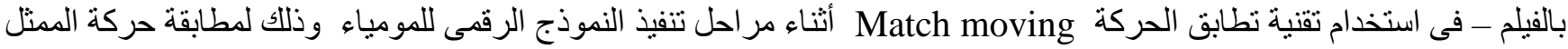

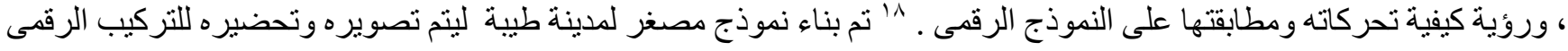

$$
\begin{aligned}
& \text { * ستنفن سومرز Stephen Sommers ( } 1962 \text { - ) : هو مخرج وكاتب سيناريو أمريكي. }
\end{aligned}
$$

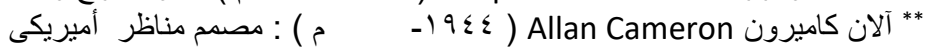

$$
\begin{aligned}
& \text { ***** جون بيرتون John Berton : هو رسام رسوم متحركة أمريكي ومشرف على المئى المؤثرات البصرية . }
\end{aligned}
$$


بياقى البيئة الرقمية ثلاثية الأبعاد فيما بعد و إضافة المؤثرات البصرية اللازمة ، أيضاً نم إضافة مؤديين كعمال فى استكمال بناء التمثال الذى يشير لأبى الهول كما في أحداث الفيلم 19

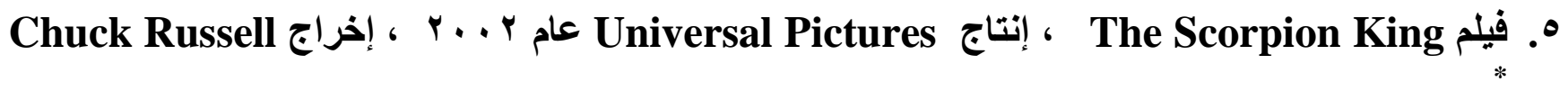

تصميم المناظر : Ed Verreaux

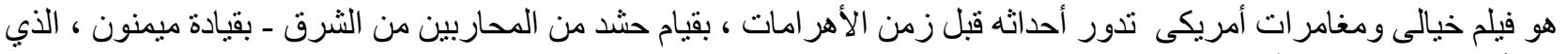

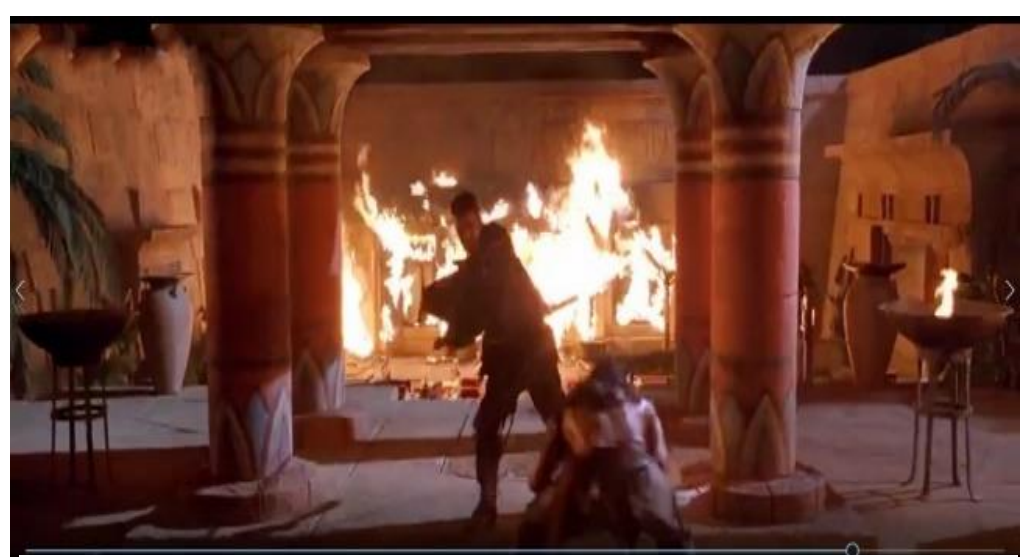

شكل 10 ، قصر الملك ميمنون كما ظهر بالفيلم ، فيلم عام2002 ـ المصدر:لقطة من الفيلم

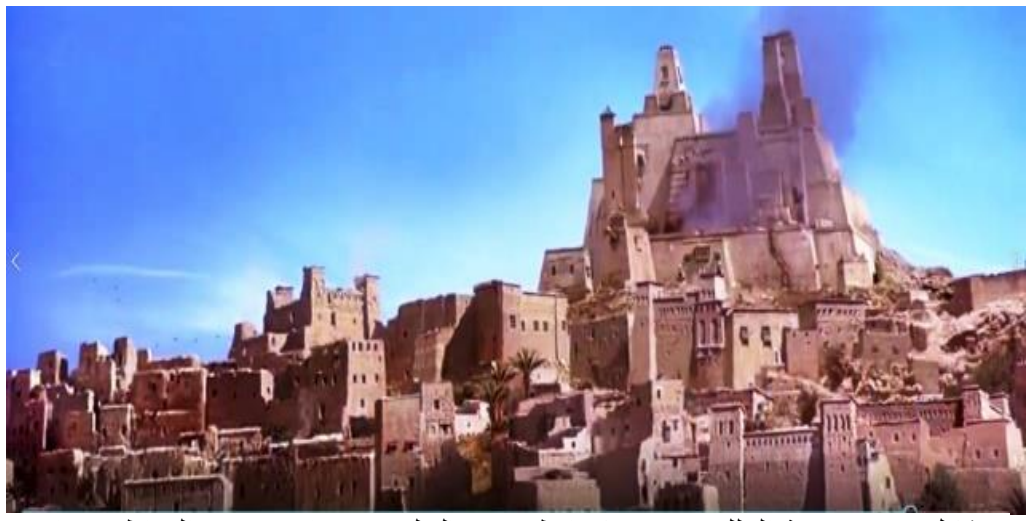

شكل 17 ، مدينة الملك ميمنون كما ظهرت بالفيلم وييدو ان مصمم المناظر قد انتقى

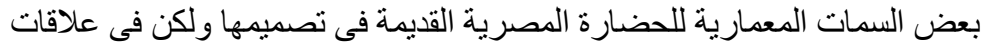

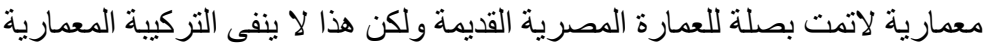

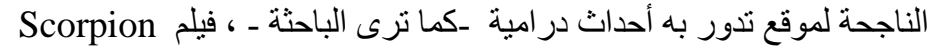

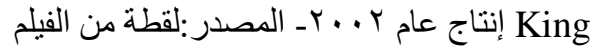

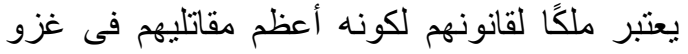

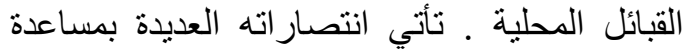

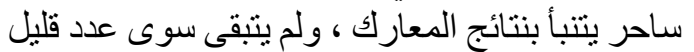

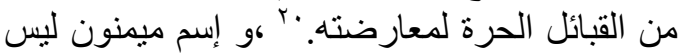

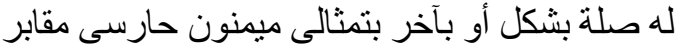

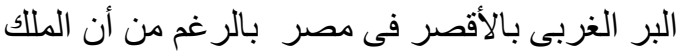

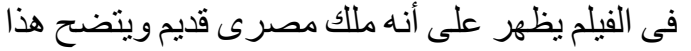
من خلال الطابع المعمارى للقصر الخاص الفه به الذئى

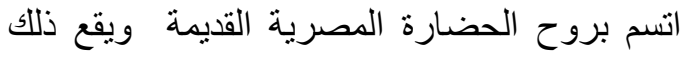

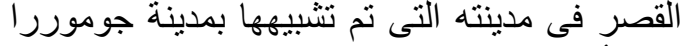

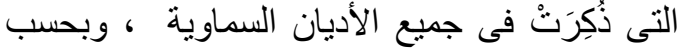
التور اهتقع على سهل نهر الأردن في المنطقة الجنوبية

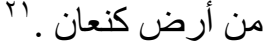
وفيما يلى تحليل لأهم مو اقع الفيلم :

\section{موقع قصر الملك ميمنون المئ}

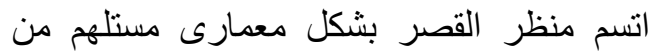
العمارة المصرية القديمة ويظهر بالبناء أعمدة تثبه

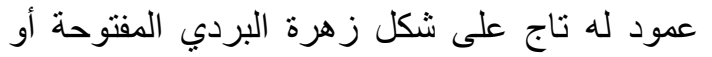

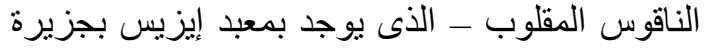
فيلة - - ولكن يشوبه الكثير من التبسيط الذى قد يجعله

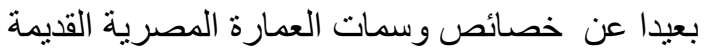
، أيضاً نسبة إرتفاع العمود وقياسها تبدو صغيرة جداً

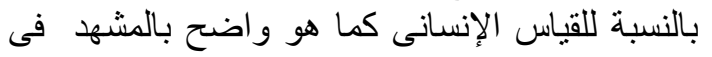
شكل 10

\section{موقع مدينة الملك ميمنون : مدينة}

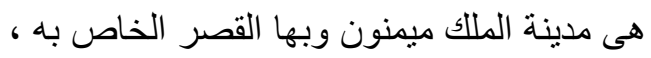

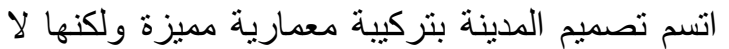
تنتمى لعمارة الحضارة المصرية بشكل أو بآخر وظهر ذلك بوضئة ولهوح فى ارتفاعات البيوت و الخصائص المعمارية المميزة لها .

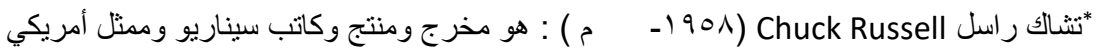

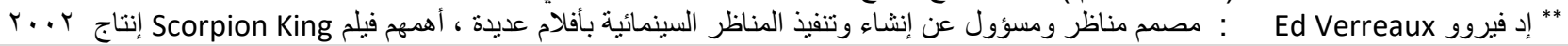




\section{SJFA}

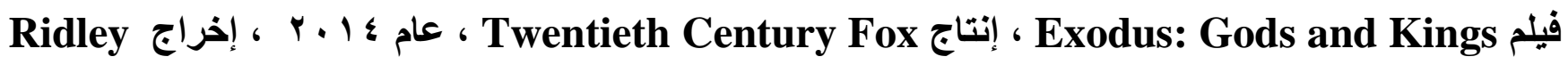
**Arthur Max تصميم المناظر للمصمم : Scott

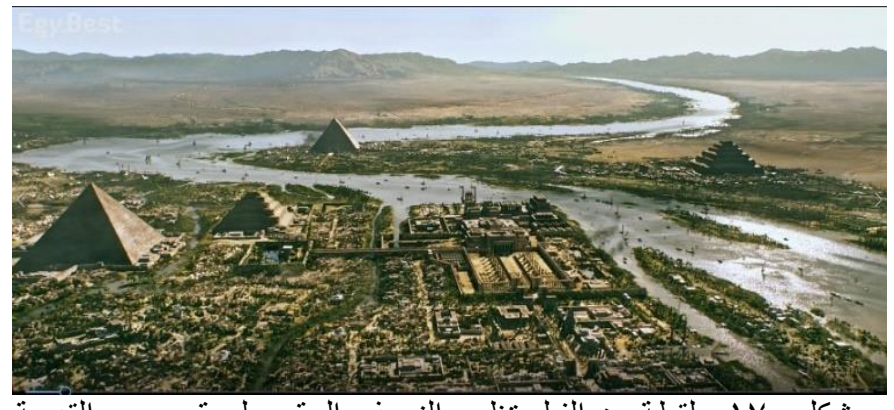
شكل IV ، لقطة من الفيلم تظهر النموذج الرقمى لموقع مصر القديمة

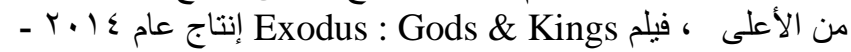
المصدر :- لقطة من الفيلم

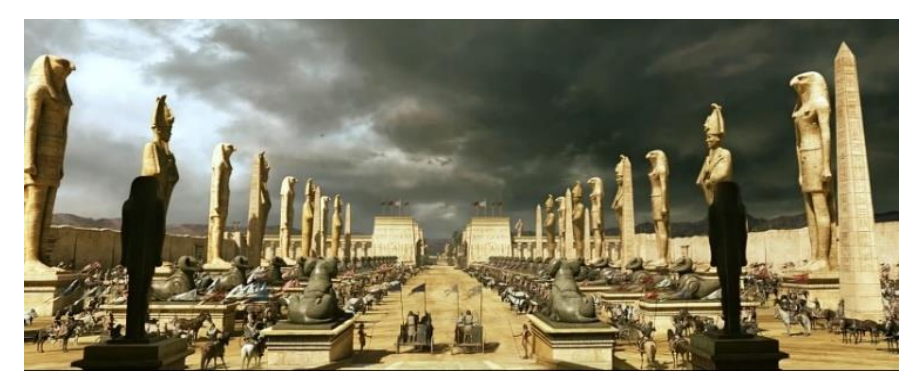

شكل 11) منظر الطريق المؤدى لقصر سيتى كما ظهر بالفيلم

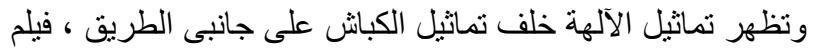
Exodus : Gods \& Kings

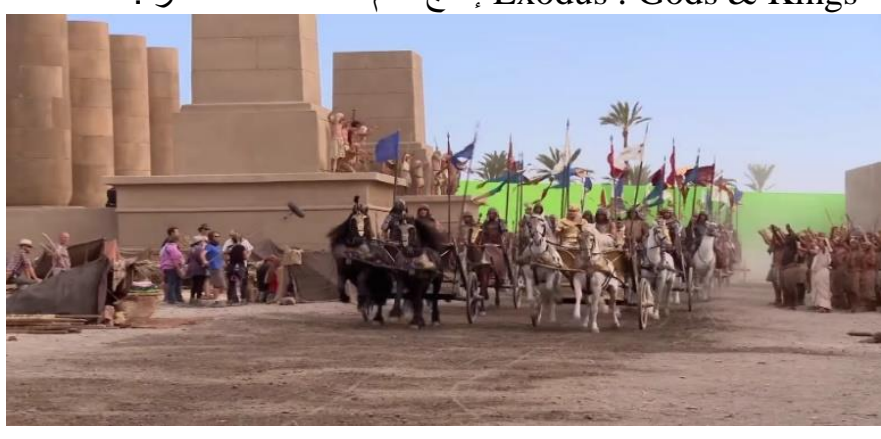

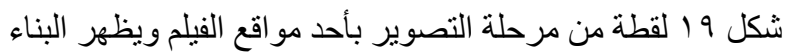

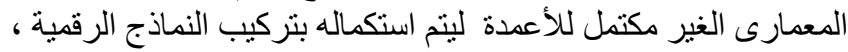

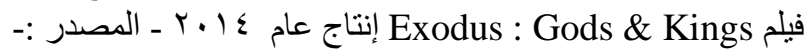
https://mattmulcahey.wordpress.com/2014/12/11/behin d-the-scenes-exodus-gods-and-kings-2014/\#jpcarousel-8682 لا تتخلاها تماثيل ضخمة للآلهة تقف بينه المه كما ظهر بالفيلم ، ربما تمت إضافتها لتشير إلى الآلهة المتعددة للمصريين القدماء .

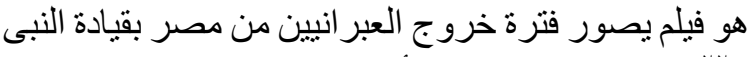

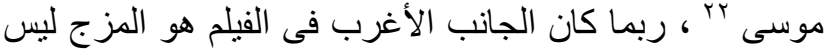

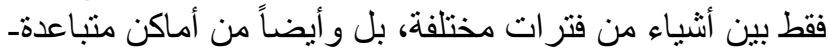

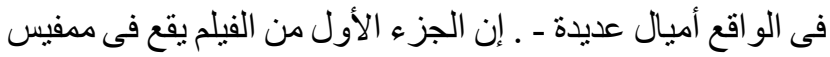
التى كانت فى الحقيقة العاصمة الإدارية لمصر ألثاء الثناء فترة كبيرة

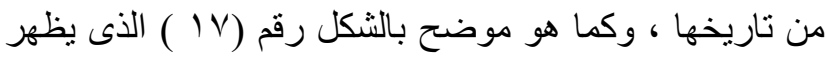

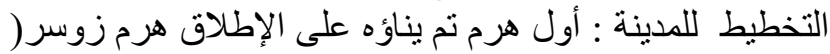

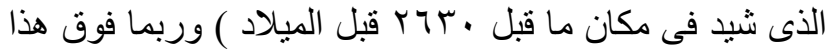

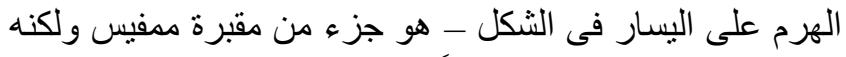

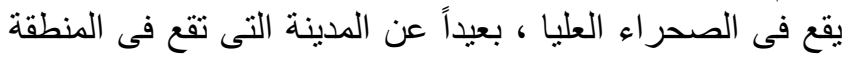

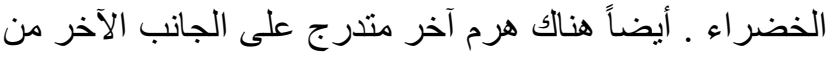

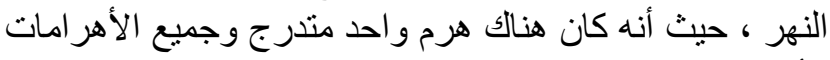

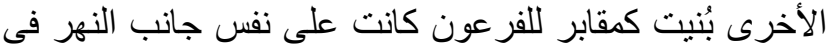

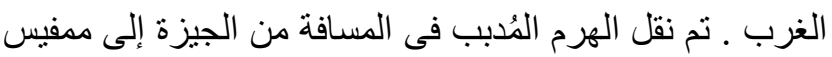

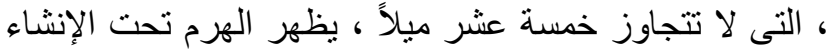

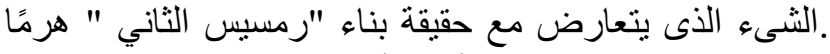

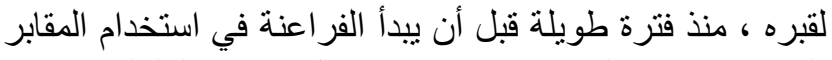

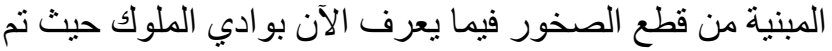

دفنه.

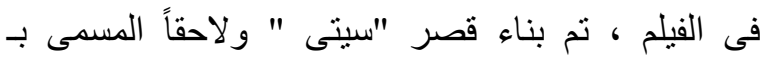

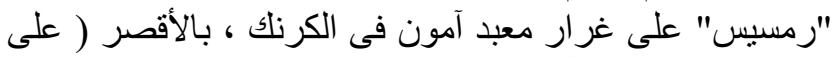

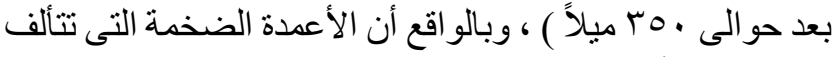

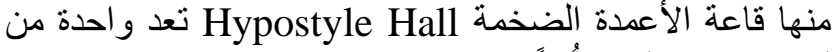

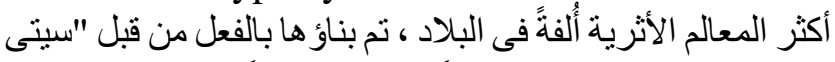

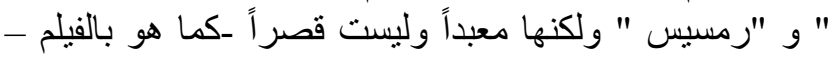

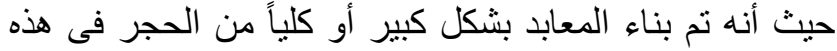

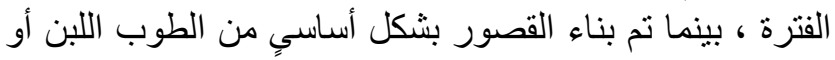

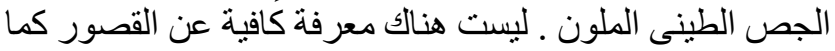

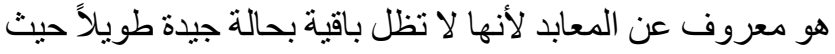

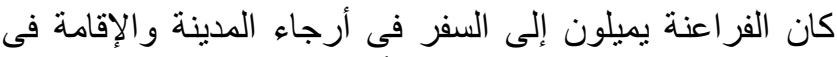

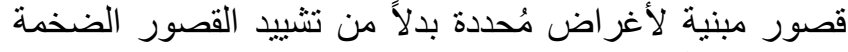

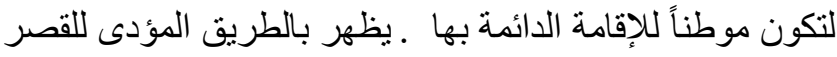

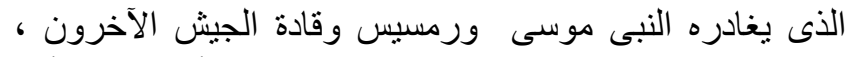

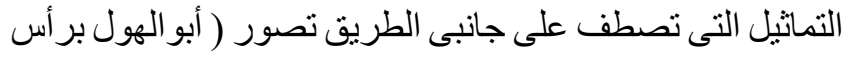

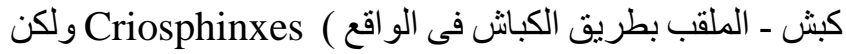

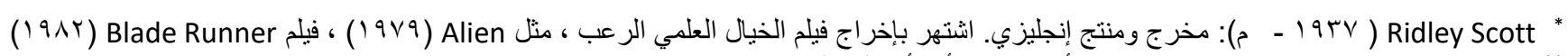

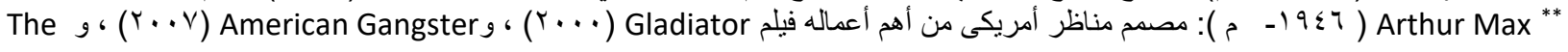




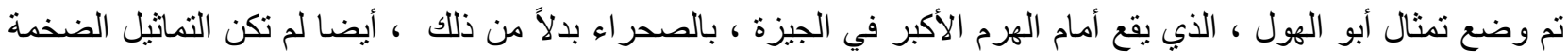

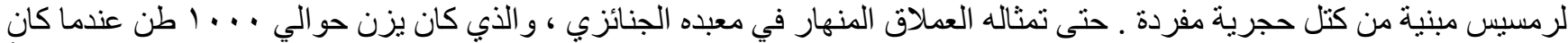

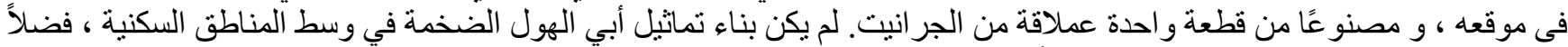

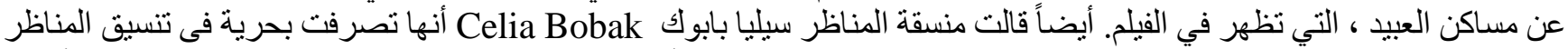

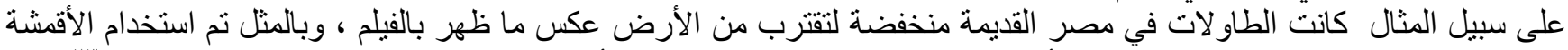

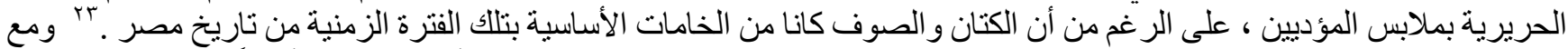

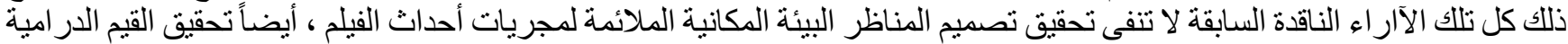

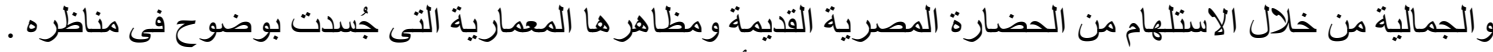

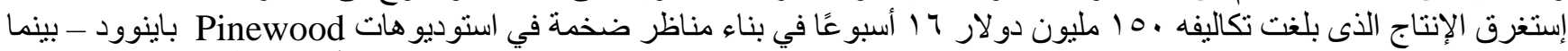

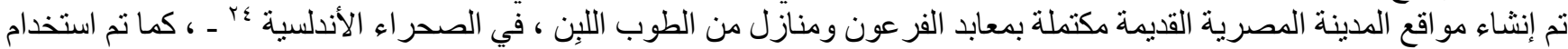

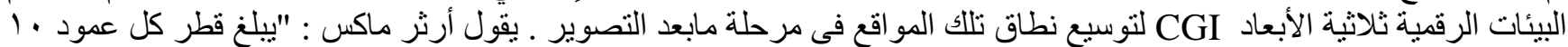

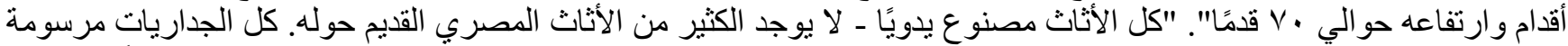

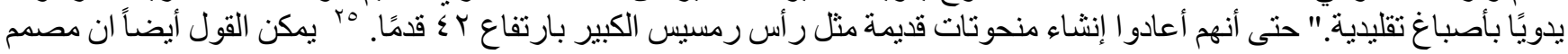

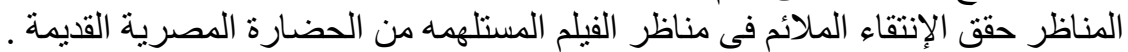

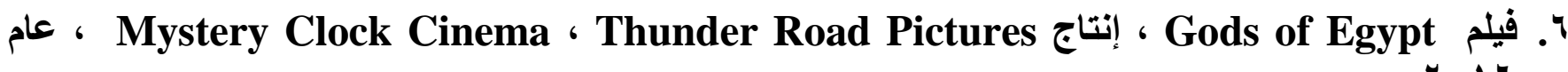
$r+17$

إخراج أليكس بروياس Alex Proyas * * تصميم المناظر للمصمم أوين باتيرسون Owen Paterson ،

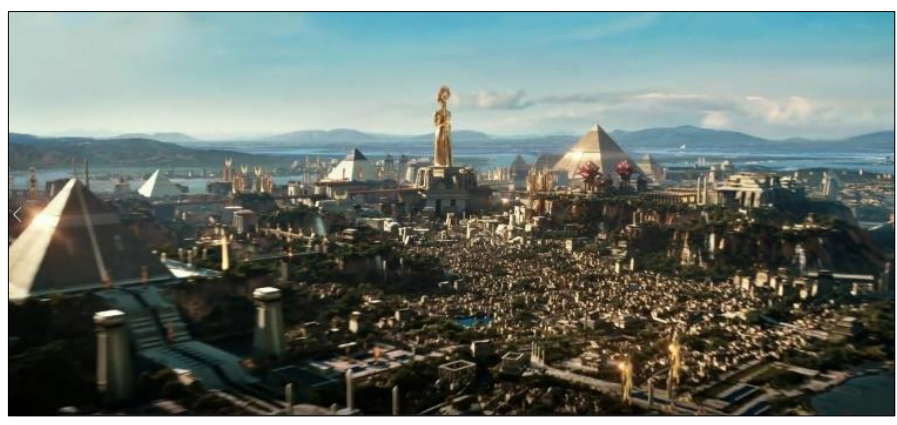

شكل · ، لقطة من الفيلم تظهر النموذج الرقمى لموقع مصر القديمة

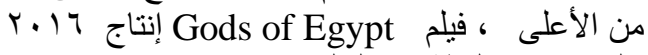
المصدر :- لقطة من الفيلم

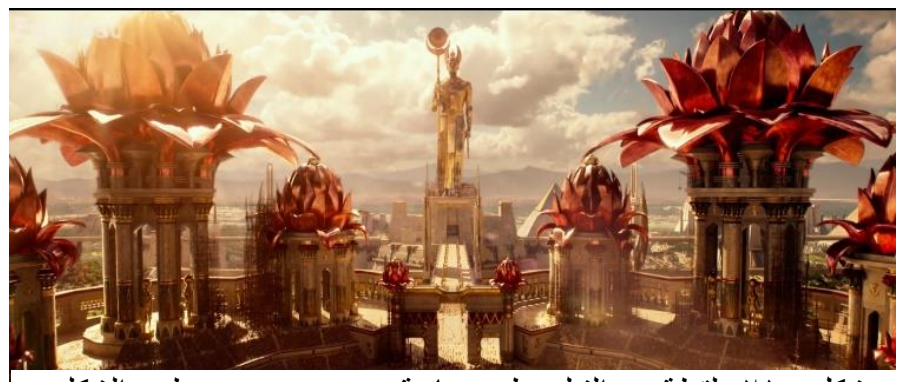

شكل اب ا، لقطة من الفيلم تظهر ساحة تتويج حورس ويظهر الشكل

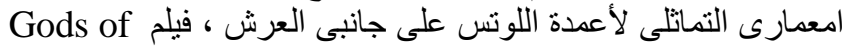
Egypt
يعد هذا الفيلم خيالياً إلى حدٍ كبير ، تدور أحداثه حول "

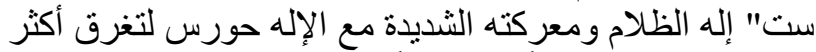

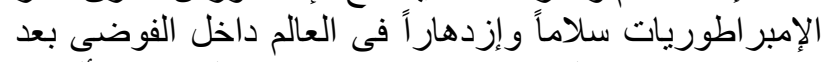
سيطرة "ست" على عرش مصر وكانت الملابس بألو انها

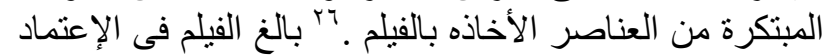

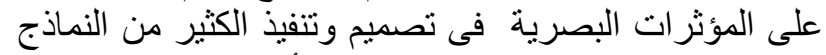
الرقمية و المؤثرات الخاصة الثى الذى الظى أظهر كثير من صور

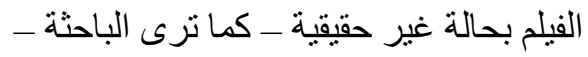

تميزت أحداث الفيلم بتحول نماذج الآلهه لكائنات أسطورية لايها قدرات خارقة كامنة بداخلها وتظهر وقت التحول و التى تم

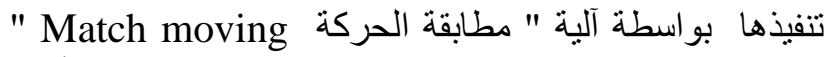
وهى تطابق حركة المؤدى مع النموذج الرقمى الثلاثى الأبعاد

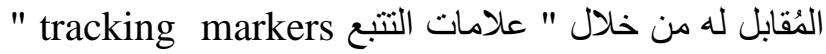

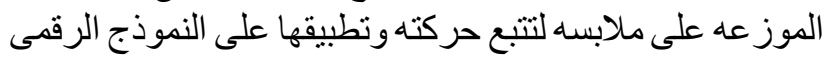
ثلاثى الأبعاد ، و تم استخدام تقنية لتكبير مقياس نماذج الآلكها عن المقياس الإنسانى - 9 أقدام- ،مع الاستعانه بآلية المنظور

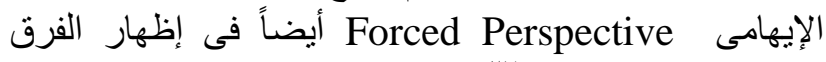

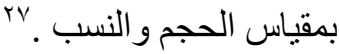

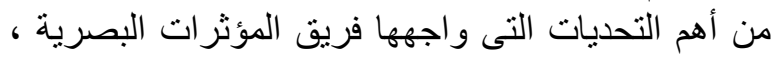

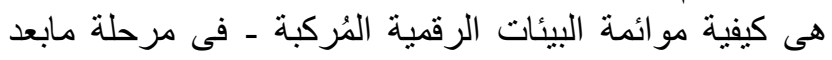

Gods أليكس بروياس Alex Proyas *

(Y. (T) of Egypt

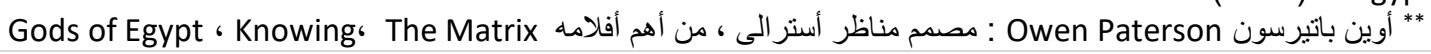


دالتصوير - مع فارق مقياس الحجم بين المؤديين لنماذج الآلهة والمؤديين للنماذج البشرية فى نفس توقيث تصوير أداء الحدث الدرامى .

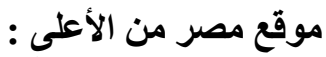

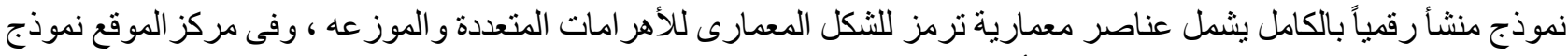

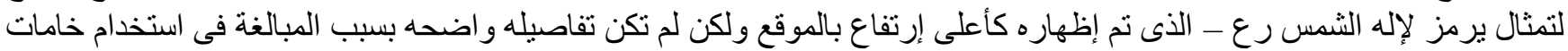

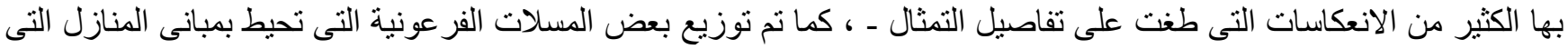

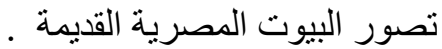

\section{موقع السوق بأحد الأحياء المصرية القديمة :}

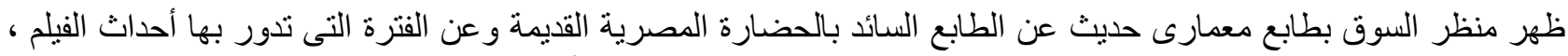
حيث استلهم مصدم المناظر الطابع المعمارى من لوحات المستشرقين التى وصفت الأحياء المصرية فى لوحات لوحات زيتية ملونة كما هو

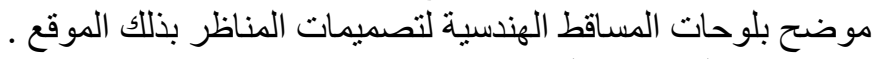
موقع الساحة الإحتفالية بتتويج حورس لعرش العند مصر:

يُعَّ من أهم المظاهر المعمارية التى تصدرت الموقع الأعمدة شـاهقة الإرتفاع المستلهمة من أعمدة اللوتس بالحضارة المصرية القديمة

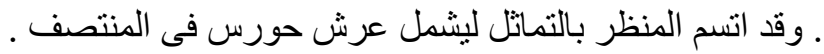
: V

بالر غم من ثر اء وتتوع المظاهر المعمارية للحضارة المصرية القديمة ، لم تتجح السينما الأميريكية فى تقديم صورة تتناسب و عظمتها

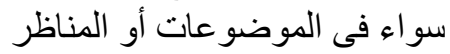
اعتمدت السينما الأميريكية فى أفلامها القوات القيلة عن الحضارة المصرية القديمة على محاكاة المواقع المعمارية والآثار الثهيرة

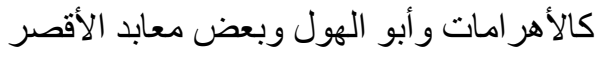

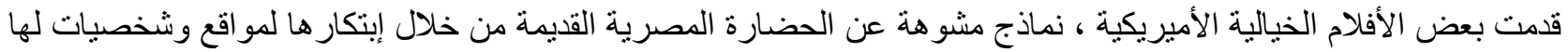

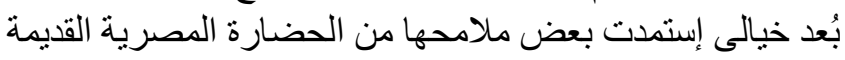

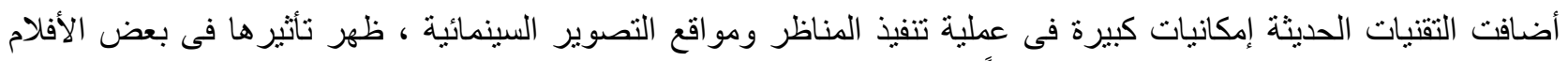
المستلهمة من الحضارة المصرية القديمة مؤخر أ الميات

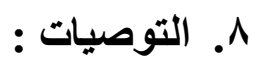

تشجيع شركات الإنتاج المصرية على إنتاج أفلام مُستلهمة من الحضارة المصرية القديمة لقيمتها التاريخية والثقافية ، و لإعادة

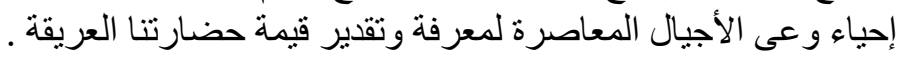

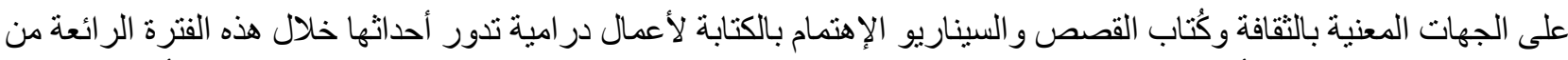

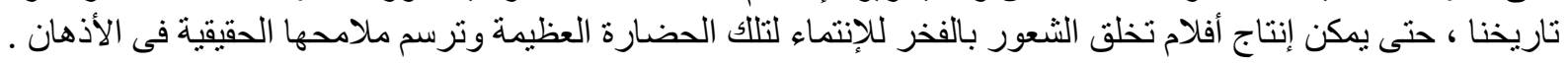
على الجهات المعنية الإهتمام بتدريس التاريخ المصرى القديم للعمارة والفنون فى مصر القديمة .

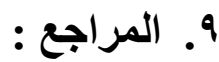

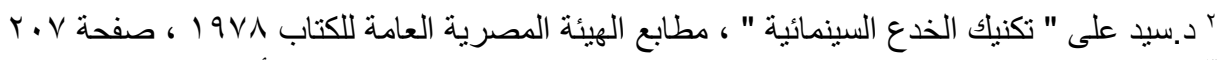

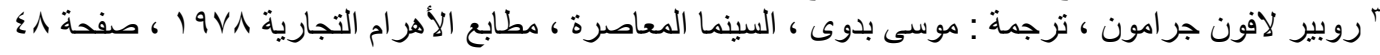

${ }^{4}$ From Wikipedia, the free encyclopedia , The Ten Commandments (1956 film), last edited on 17 /10/2020,

${ }^{5}$ Movie Locations "The Ten Commandments | 1956 " ( https://www.movie-locations.com/movies/t/TenCommandments-1956.php ) 10/10/2020

${ }^{6}$ Lourdes Font, 1963 - MANKIEWICZ, CLEOPATRA , Last updated Mar 16, 2018 | Published on Aug 27, 2017 , ( https://fashionhistory.fitnyc.edu/1963-mankiewicz-cleopatra/ ) , 29/8/2020 
${ }^{7}$ Cathy Whitlock , The Set Designs of Cleopatra, Elizabeth Taylor's Classic Movie, December 31, 2012 (https://www.architecturaldigest.com/gallery/cleopatra-elizabeth-taylor-set-design-50th-anniversary-cannesslideshow ), 28/8/2020

${ }^{8}$ Craig Leask , CLEOPATRA (1963): THE SETS BEHIND THE EPIC , 24/92018

(https://footeandfriendsonfilm.com/2018/09/24/cleopatra-1963-the-sets-behind-the-epic/ ) , 28/8/2020

${ }^{9}$ Domingo Lizcano , Joseph Natanson memoirs of Clepopatra(1963) , 19 December, 2016 (

http://bigerboat.com/indexfx/?p=3777 ) 12/9/2010

${ }^{10}$ Magdalena Julianna Magenta, Magdalena Julianna Magenta, ANCIENT SOURCES FOR FILM SCENOGRAPHY "KLEOPATRA" JOSEPH MANKIEWICZ, MA thesis, FACULTY OF HISTORICAL AND SOCIAL SCIENCES, CARDINAL STEFAN WYSZYŃSKI UNIVERSITY IN WARSAW, 2017, p. 3 纟

${ }^{11}$ From Wikipedia, the free encyclopedia , Stargate (film), ( https://en.wikipedia.org/wiki/Stargate_(film) ), $4 / 9 / 2020$

${ }^{12}$ Catawiki , Iconic 'Stargate' Concept Art On Auction at Catawiki , ( https://www.catawiki.com/stories/5139iconic-stargate-concept-art-on-auction-at-catawiki ) , 4/9/2020

${ }^{13}$ PAUL WILLISTEIN, 29/10/1994 , FILMING 'STARGATE' A GREAT ADVENTURE FOR KURT RUSSELL AND JAMES SPADER , , The Morning Call ( https://www.mcall.com/news/mc-xpm-1994-10-29-3008800-story.html ) $12 / 9 / 2020$

${ }^{14}$ ThePropKing , Stargate (1994) - Behind the Scenes - How the Stargate was made, $1 / 4 / 2011$,Youtube (https://www.youtube.com/watch?v=Bj-EPB25ROQ\&t=174s ) 22/9/2020

${ }^{15}$ ThePropKing , Stargate (1994) - Behind the Scenes - How Ra's tomb was made , 10/4/2011, Youtube ( https://www.youtube.com/watch?v=u9Fd4t4XMdU ) 22/9/2020

${ }^{16}$ Toolbox , Shoot , Stargate Studios creates real time virtual environments using Blackmagic Design , 18/5/ 2020 , ( https://www.shootonline.com/node/84540 ) 20/9/2020

${ }^{17}$ Stargate Studios , 7/7/2020, Virtual Movie Production Comes to Australia

Global Leader Stargate Studios to Open at AMPCO Studios in Adelaide, Cision Pr newswire , (

https://www.prnewswire.com/news-releases/virtual-movie-production-comes-to-australia-301088776.html ) 22/9/2020

${ }^{18}$ From Wikipedia, the free encyclopedia , The Mummy (1999 film) , ( https://en.wikipedia.org/wiki/The Mummy (1999 film ) , 6/9/2020

${ }^{19}$ Authorlan Failes, Before you see Tom Cruise's 'The Mummy', re-visit the digital make-up, mocap and other VFX innovations from the 1999 film , vfxblog Visual effects and animation journalist lan Failes(

https://vfxblog.com/2017/06/05/the-mummy-1999-vfx/) June 5, 2017 , 6/9/2020

${ }^{20}$ From Wikipedia, the free encyclopedia , The Scorpion King , ( https://en.wikipedia.org/wiki/The Scorpion King ) 20/9/2020

${ }^{21}$ From Wikipedia, the free encyclopedia , Sodom and Gomorrah ( https://en.wikipedia.org/wiki/Sodom and Gomorrah ) 20/9/2020

${ }^{22}$ From Wikipedia, the free encyclopedia , Exodus: Gods and Kings , ( https://en.wikipedia.org/wiki/Exodus: Gods and Kings ) , 12/9/2020

${ }^{23}$ Kristin Thompson and David Bordwell , EXODUS: GODS AND KINGS and the myth of authenticity , Observations on Film art ( http://www.davidbordwell.net/blog/2015/01/11/exodus-gods-and-kings-and-themyth-of-authenticity/ ) Last Modified: Sunday | July 26, 2015 @ 20:47 ,searched 24/9/2020

${ }^{24}$ Casa Oleo , EXODUS: GODS \& KINGS, FILMED IN ANDALUCIA, OPENS IN SPAIN , December 7, 2014 ( http://www.casaolea.com/blog/exodus-gods-kings-filmed-andalucia-opens-spain ) 20,September 2020

${ }^{25}$ Carolyn Giardina , 25 November 2014 , Oscars: How Ancient Egypt's Royal Palace Was Created for 'Exodus' and 6 More Film Set Secrets, The Hollywood Reporter ( https://www.hollywoodreporter.com/behindscreen/oscars-how-ancient-egypts-royal-751929 ) 20 September 2010 . 


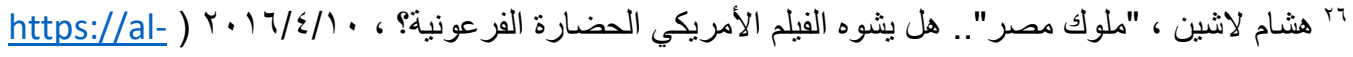
( ain.com/article/116676?fbclid=IwAR10kZUlyHARqzmX42nUuULSF hUA219g-px HGk7uFkKKrGtHGaq3v24SM $r \cdot r \cdot / 9 / 1$.

${ }^{27}$ Vincent Frei , GODS OF EGYPT: ERIC DURST - OVERALL VFX SUPERVISOR , The Art of VFX , 4 /4/ 2016 ( https://www.artofvfx.com/gods-egypt-eric-durst-overall-vfxsupervisor/?fbclid=IwAR3OHgxKLXLxshxfIWW2F1t-Dt2-j4Y2YVMUhPbxQ7jz2naViLBSakJ4P5U ) 11/9/2020

${ }^{28}$ Fxphd, Using every trick in the VFX book: Gods of Egypt , ( https://www.fxphd.com/fxblog/using-every-trickin-the-vfx-book-gods-of-egypt/ ) 11/9/2020 . 


\section{الاورية العلمية لكلية الفنون الجميلة - جامعة الإسكندرية}

SJFA

\title{
Ancient Egyptian Civilization in American Cinema since the Second Half of the $20^{\text {th }}$ Century
}

(A Case Study on Sets \& Locations)

R. M. Hilal

\begin{abstract}
From time to time, American motion picture production companies produce films whose events revolve around or whose themes are inspired by the ancient Egyptian civilization, and these films bear special features due to the impact of the Egyptian civilization on the history of humanity and in its architectural and aesthetic aspects that are clearly visible in the remaining architectural and artistic effects of the Egyptian civilization The ancient, which is the largest compared to the effects of other ancient civilizations, also for its most impressive views and indicative of scientific and artistic progress, especially in the field of architecture, those factors and characteristics that were the source of passion and inspiration for the authors and filmmakers to embody them in American films, as some of their themes were derived from Old Testament stories or stories Kings and rulers or some fictional novels that tried to take advantage of the mystery and prestige of their architecture and art. The designs of the scene designers in these films varied between trying to commit to the historical and inspiring the spirit of the Egyptian civilization and its general features in proportion to the events of the film and its dramatic requirements, and this study provides an analysis of the scenes and filming locations of the most important American cinematic films that were inspired by the ancient Egyptian civilization. Since the beginning of the fifties of the last century until now, we find - through that study - that despite the richness and diversity of the architectural aspects of the ancient Egyptian civilization, American cinema has not succeeded in presenting an image commensurate with its greatness, whether in subjects or scenes, also the adoption of American cinema in its films The few about the Egyptian civilization simulate the archaeological sites and famous monuments such as the pyramids, the Sphinx and some of the Luxor temples, in spite of this it presented distorted models of the ancient Egyptian civilization through its creation of sites and characters with an imaginary dimension, which obliterated the basic features and features of the ancient Egyptian civilization. And finally, taking advantage of modern technologies that have added great potential in the process of executing scenes and performance sites in American cinema films.
\end{abstract}

KEYWORDS: Format, Introduction, fonts, headings, figures, equations. 